\title{
A common limit of super Liouville theory and minimal models
}

\author{
Stefan Fredenhagen \\ Max-Planck-Institut für Gravitationsphysik, Albert-Einstein-Institut \\ D-14424 Golm, Germany \\ E-mail: Stefan.fredenhagen@aei.mpg.de
}

\section{David Wellig}

Institut für Theoretische Physik, ETH Zürich

CH-8093 Zürich, Switzerland

E-mail: welligd@gmx.net

ABSTRACT: We show that $N=1$ supersymmetric Liouville theory can be continued to central charge $c=3 / 2$, and that the limiting non-rational superconformal field theory can also be obtained as a limit of supersymmetric minimal models. This generalises a result known for the non-supersymmetric case. We present explicit expressions for the three-point functions of bulk fields, as well as a set of superconformal boundary states. The main technical ingredient to take the limit of minimal models consists in determining analytic expressions for the structure constants. In the appendix we show in detail how the structure constants of supersymmetric and Virasoro minimal models can be rewritten in terms of Barnes' double gamma functions.

Keywords: Conformal Field Models in String Theory, Conformal and W Symmetry. 


\section{Contents}

1. Introduction 1

2. Limit of super minimal models 3

2.1 Preliminaries 3

2.2 Spectrum 司

2.3 Operator product expansion

2.4 Two-point functions 6

2.5 Three-point functions

2.6 One-point functions on the upper half plane

3. Limit of super Liouville theory 9

3.1 Preliminaries 9

3.2 Two- and three-point functions: Neveu-Schwarz sector 10

3.3 Two- and three-point functions: Ramond sector 12

3.4 One-point functions on the upper half plane 14

4. Summary and outlook 15

A. Structure constants in terms of Barnes' double gamma functions 16

A.1 Virasoro minimal models 16

A.2 Superconformal minimal models

B. Special functions 22

G. Fusion rules from Liouville 23

\section{Introduction}

The classification of conformal theories for a given central charge is a hard and in general maybe unmanageable task. For unitary theories, the classification has only been achieved for central charge $c<1$ resulting in the well known ADE series of minimal models [1, 2]. One might hope that the problem still remains tractable for the limiting value $c=1$. In [3] the moduli space of $c=1$ models based on the free boson was presented. At some points of the moduli space, the theory is rational, and it was argued in [4] that these are already all rational theories at $c=1$. That there are further non-rational models that cannot be obtained from the free boson was shown by Runkel and Watts [5] by explicitly constructing a new not even quasi-rational theory at $c=1$. 
Runkel and Watts considered the unitary minimal models and defined a new theory as the limit of minimal models when the central charge approaches $c=1$. They provided an explicit expression for the structure constants, and gave strong evidence that the resulting non-rational theory is crossing-symmetric. Later in [6], Schomerus considered a continuation of Liouville theory to central charge $c=1$, and found that it agrees with the Runkel-Watts theory. In [5], the authors also constructed boundary states for this theory as a limit of minimal model boundary states (see also [7]). It was then understood in [8] that these boundary states can be obtained from the ZZ boundary states [9] in Liouville theory. There, also the limit of the FZZT boundary states [10, 11] was constructed and it was shown how these are obtained from the minimal model side.

In this paper we shall construct a similar limit of minimal models in the $N=1$ supersymmetric case. The result is a non-rational theory at the critical central charge $c=\frac{3}{2}$ (below this value all unitary theories fall into the rational minimal model series [12]). It is the first example of a unitary superconformal theory at $c=\frac{3}{2}$ that is not part of the moduli space of supersymmetric $c=\frac{3}{2}$ theories that are obtained from a free boson and a free fermion [13]. As in the bosonic case, the limiting theory can also be obtained from $N=1$ supersymmetric Liouville theory.

The main technical ingredient in the computation of Runkel and Watts was the unpublished observation of Dotsenko that the structure constants of minimal models can be written as analytic functions depending on a certain combination of the field labels. In appendix A we present a derivation of this fact. This then allows the continuation of the structure constants to the limiting theory.

In the $N=1$ supersymmetric case, the minimal model structure constants (in the Neveu-Schwarz sector) have been determined in [14] (see also [15]). We derive here an analytic expression for these structure constants by rewriting them in terms of Barnes' double gamma functions, special functions that naturally appear in Liouville theory. The limit can then be taken in close analogy to the derivation in [5]. We shall provide the bulk structure constants in the Neveu-Schwarz sector, as well as the bulk one-point functions for a discrete family of boundary conditions.

The $N=1$ Liouville three-point functions have been computed in [16, 17] (see also [18, 19]). Barnes' double gamma functions from which they are built become singular in the limit $c \rightarrow \frac{3}{2}$. To deal with this singularity, we make use of asymptotic expressions for Barnes' double gamma functions that have been derived in [6, 8]. This allows us to write the three-point function as a product of a non-singular analytic factor, and a factor that becomes a discontinuous but finite step function, which implements fusion rules. The result coincides with the expressions we obtain from the minimal models. For a discrete family of boundary conditions we evaluate the bulk one-point function and show that it agrees with the minimal model limit.

The paper is organised as follows. In section 2 we determine the limit of supersymmetric minimal models, in section 3 the limit of super Liouville theory is computed and compared to the minimal model result. Section 4 summarises our results and discusses the open problems and future directions. Appendix A contains the derivation of the main structural results, namely the rewriting of the (super) minimal model structure constants 
in terms of Barnes' double gamma functions. Appendix B collects some information on the special functions that are frequently used in the paper. The last appendix $\mathrm{C}$ explicitly shows how the fusion rules pop up from the limit of Liouville theory.

\section{Limit of super minimal models}

\subsection{Preliminaries}

The unitary, $N=1$ supersymmetric minimal models can be labelled by an integer $p \geq 3$, the central charge is given by 20

$$
c=\frac{3}{2}\left(1-\frac{8}{p(p+2)}\right) .
$$

In each model there are a finite number of primary fields $\phi_{r s}$, which are parameterised by two integers $(r, s)$ with $1 \leq r \leq p-1$ and $1 \leq s \leq p+1$. The combinations $(r, s)$ and $(p-r, p+2-s)$ label the same field. The conformal weight of a primary field $\phi_{r s}$ is

$$
h_{r s}=\frac{((p+2) r-p s)^{2}-4}{8 p(p+2)}+\frac{1}{32}\left(1-(-1)^{r-s}\right) .
$$

The fields $\phi_{r s}$ with $r-s$ even belong to the Neveu-Schwarz (NS) sector, the fields with $r-s$ odd to the Ramond (R) sector. In the Neveu-Schwarz sector, to each superconformal primary field $\phi_{r s}$, there is a superdescendant field $\tilde{\phi}_{r s}$, which is primary with respect to the bosonic subalgebra. Generically, it has conformal weight $\tilde{h}_{r s}=h_{r s}+\frac{1}{2}$ (the exception being the vacuum whose superdescendant is the supercurrent at conformal weight $\frac{3}{2}$ ).

\subsection{Spectrum}

We are interested in the limit of large parameter $p$ when the central charge approaches $c=\frac{3}{2}$. To understand how the conformal weights of the primary fields behave in that limit, it is instructive to rewrite the conformal weight as

$$
\begin{aligned}
h_{r s}^{\mathrm{NS}} & =\frac{d_{r s}^{2}-d_{11}^{2}}{8 t} \\
h_{r s}^{\mathrm{R}} & =\frac{d_{r s}^{2}}{8 t}+\frac{c}{24},
\end{aligned}
$$

with $d_{r s}=r-s t$ and $t=\frac{p}{p+2}$. Because of field identifications, we can restrict attention to labels $(r, s)$ satisfying $r \geq s t$ (where $r=s t$ can only be satisfied for the Ramond ground state, which exists for even $p$ ). Let us rewrite

$$
d_{r s}=(r-s)+\frac{2}{p+2} s,
$$

where the first term is an even (odd) integer in the Neveu-Schwarz (Ramond) sector, and the second term ranges between 0 and 2. For a given $d$ we look for $d_{r s}$ that approximate $d$ within a small interval of size $\epsilon$, so we introduce the set

$$
N(d, \epsilon)=\left\{(r, s) \mid d \leq d_{r s}<d+\epsilon\right\} .
$$


In the following we shall restrict the discussion to the Neveu-Schwarz sector. For $\epsilon$ small enough ( $d$ and $d+\epsilon$ should have the same even integer part) this set is given by

$$
N(d, \epsilon)=\left\{\left(2\left\lfloor\frac{d}{2}\right\rfloor+n, n\right) \mid\left\{\frac{d}{2}\right\}(p+2) \leq n<\left(\left\{\frac{d}{2}\right\}+\frac{\epsilon}{2}\right)(p+2)\right\} .
$$

Here, $\lfloor x\rfloor$ is the largest integer smaller or equal to $x$, and $\{x\}$ denotes the fractional part of $x, x=\lfloor x\rfloor+\{x\}$. For large $p$ the number of pairs $(r, s)$ contributing to $N(d, \epsilon)$ grows as

$$
|N(d, \epsilon)| \sim \frac{1}{2} \epsilon(p+2) .
$$

In particular we see that any value of $d$ can be approximated by the $d_{r s}$ with uniform density, i.e. the number of $d_{r s}$ per unit interval is independent of $d$,

$$
\frac{1}{\epsilon}|N(d, \epsilon)| \sim \frac{p+2}{2} .
$$

Following the prescription of [5], we define fields $\phi_{d}$ of the theory at $c=\frac{3}{2}$ by averaging over fields that lead to the same conformal weight in the limit, ${ }^{1}$

$$
\phi_{d}(z, \bar{z}):=\lim _{\epsilon \rightarrow 0} \lim _{p \rightarrow \infty} \frac{n(p)}{|N(d, \epsilon)|} \sum_{(r s) \in N(d, \epsilon)} \phi_{r s}(z, \bar{z}) .
$$

The function $n(p)$ will be chosen in the next subsection to get fields in the appropriate normalisation. Note that in the following we shall suppress the argument $\bar{z}$ in the fields $\phi$.

\subsection{Operator product expansion}

We require that in the limit the operator product expansion (OPE) of two fields $\phi_{d_{i}}$ remains finite, i.e. it should be of the form

$$
\phi_{d_{1}}\left(z_{1}\right) \phi_{d_{2}}\left(z_{2}\right)=\int \mathrm{d} d_{3} \frac{1}{\left|z_{12}\right|^{2\left(h_{1}+h_{2}-h_{3}\right)}} D\left(d_{1}, d_{2}, d_{3}\right)\left(\phi_{d_{3}}\left(z_{2}\right)+\cdots\right)+\text { superdescendants },
$$

with some function (or distribution) $D\left(d_{1}, d_{2}, d_{3}\right)$. The dots stand for contributions of Virasoro descendant fields, and $z_{12}=z_{1}-z_{2}$. From the definition (2.10) of the fields $\phi_{d}$ we get

$$
\begin{aligned}
& \phi_{d_{1}}\left(z_{1}\right) \phi_{d_{2}}\left(z_{2}\right)=\lim _{\epsilon \rightarrow 0} \lim _{p \rightarrow \infty} \frac{4 n(p)^{2}}{\epsilon^{2} p^{2}} \sum_{\left(r_{i} s_{i}\right) \in N\left(d_{i}, \epsilon\right)} \phi_{r_{1} s_{1}}\left(z_{1}\right) \phi_{r_{2} s_{2}}\left(z_{2}\right)+\cdots \\
& =\lim _{\epsilon \rightarrow 0} \lim _{p \rightarrow \infty} \frac{4 n(p)^{2}}{\epsilon^{2} p^{2}} \sum_{\left(r_{i} s_{i}\right) \in N\left(d_{i}, \epsilon\right)} \sum_{r_{3}, s_{3}} \mathcal{N}_{r_{1} r_{2}}^{(p)} r_{3} \mathcal{N}_{s_{1} s_{2}}^{(p+2) s_{3}} \\
& \times\left(\frac{\delta_{2}(k+l)}{\left|z_{12}\right|^{2\left(h_{r_{1} s_{1}}+h_{r_{2} s_{2}}-h_{\left.r_{3} s_{3}\right)}\right.}} D_{\left(r_{1} s_{1}\right)\left(r_{2} s_{2}\right)}^{\mathrm{NS}}{ }^{\left(r_{3} s_{3}\right)} \phi_{r_{3} s_{3}}\left(z_{2}\right)\right. \\
& \left.+\frac{\delta_{2}(k+l+1)}{\left|z_{12}\right|^{2\left(h_{r_{1} s_{1}}+h_{r_{2} s_{2}}-\tilde{h}_{\left.r_{3} s_{3}\right)}\right.}} \tilde{D}_{\left(r_{1} s_{1}\right)\left(r_{2} s_{2}\right)}^{\mathrm{NS}}\left(r_{3} s_{3}\right) \tilde{\phi}_{r_{3} s_{3}}\left(z_{2}\right)\right)+\cdots .
\end{aligned}
$$

\footnotetext{
${ }^{1}$ for a discussion of some aspects of limits of superminimal models where instead of $d$ the labels $(r, s)$ are fixed see 21
} 
We used here the operator product expansion (A.19) for minimal model fields, which is stated in appendix A. In the OPE the fusion rules enter which can be expressed in terms of the fusion rules $\mathcal{N}^{(p)}$ (given in (A.3)) of the $s u(2)$ WZW model. $\delta_{2}(n)$ is defined to be 1 for $n$ even, and 0 for $n$ odd.

The important insight is that the structure constants only depend on the combinations $d_{r_{i} s_{i}}=r_{i}-t s_{i}$, and that this dependence is analytic. This result is derived in appendix A, the final expressions for the structure constants are given in (A.29) and (A.31). It can easily be seen that the expressions have well-defined limits $D_{d_{1} d_{2}}^{\mathrm{NS}} d_{3}, \tilde{D}_{d_{1} d_{2}}^{\mathrm{NS}} d_{3}$ as $p \rightarrow \infty$,

$$
D_{d_{1} d_{2}}^{\mathrm{NS}} d_{3}=\left[\frac{\Upsilon\left(1-\frac{\tilde{d}}{2} \mid 1\right) \Upsilon\left(1-\frac{\tilde{d}_{1}}{2} \mid 1\right) \Upsilon\left(1-\frac{\tilde{d}_{2}}{2}\right) \Upsilon\left(1-\frac{\tilde{d}_{3}}{2} \mid 1\right)}{\Upsilon(1 \mid 1) \Upsilon\left(1-\frac{d_{1}}{2} \mid 1\right) \Upsilon\left(1-\frac{d_{2}}{2} \mid 1\right) \Upsilon\left(1-\frac{d_{3}}{2} \mid 1\right)}\right]^{2},
$$

and

$$
\tilde{D}_{d_{1} d_{2}}^{\mathrm{NS}} d_{3}=\frac{1}{h_{3}} \frac{\Upsilon\left(\frac{1}{2}-\frac{\tilde{d}}{2} \mid 1\right) \Upsilon\left(\frac{3}{2}-\frac{\tilde{d}}{2} \mid 1\right) \prod_{i} \Upsilon\left(\frac{1}{2}-\frac{\tilde{d}_{i}}{2} \mid 1\right) \Upsilon\left(\frac{3}{2}-\frac{\tilde{d}_{i}}{2} \mid 1\right)}{\left[\Upsilon(1 \mid 1) \Upsilon\left(1-\frac{d_{1}}{2} \mid 1\right) \Upsilon\left(1-\frac{d_{2}}{2} \mid 1\right) \Upsilon\left(1-\frac{d_{3}}{2} \mid 1\right)\right]^{2}} .
$$

Here, $2 \tilde{d}=d_{1}+d_{2}+d_{3}$ and $\tilde{d}_{i}=\tilde{d}-d_{i}$. The functions $\Upsilon(x \mid b)$ are special combinations of Barnes' double gamma functions (see appendix B).

The only thing left to consider are the fusion rules. For given $\left(r_{i}, s_{i}\right) \in N\left(d_{i}, \epsilon\right)$, $i=1,2$, we shall analyse which labels $\left(r_{3}, s_{3}\right)$ appear in the sum above due to the fusion rules $\mathcal{N}^{(p)} \mathcal{N}^{(p+2)}$. The label $\left(r_{3}, s_{3}\right)$ appears if (up to corrections of order $\epsilon$ or $1 / p$ )

$\left|\left\{\frac{d_{1}}{2}\right\}-\left\{\frac{d_{2}}{2}\right\}\right|<\left\{\frac{d_{3}}{2}\right\}<\min \left(\left\{\frac{d_{1}}{2}\right\}+\left\{\frac{d_{2}}{2}\right\}, 2-\left\{\frac{d_{1}}{2}\right\}-\left\{\frac{d_{2}}{2}\right\}\right)$ and $s_{1}+s_{2}+s_{3}$ odd .

The condition on the $s_{i}$ leads to the conclusion that for given $\left(r_{1}, s_{1}\right)$ and $\left(r_{2}, s_{2}\right)$ only one half of the labels in $N\left(d_{3}, \epsilon\right)$ appear if $d_{3}$ satisfies the inequality in 2.15). (Note that here we allow also for negative $d_{3}$, the definition of $N\left(d_{3}, \epsilon\right)$ can be extended to this case in an obvious way.) As we sum over $\left(r_{1}, s_{1}\right) \in N\left(d_{1}, \epsilon\right)$ and $\left(r_{2}, s_{2}\right) \in N\left(d_{2}, \epsilon\right)$, each label $\left(r_{3}, s_{3}\right)$ in $N\left(d_{3}, \epsilon\right)$ appears with multiplicity $\frac{1}{2}\left|N\left(d_{1}, \epsilon\right)\right|\left|N\left(d_{2}, \epsilon\right)\right|$.

The condition on $k+l$ can be reformulated in terms of the integer parts $\left\lfloor\frac{d_{i}}{2}\right\rfloor$,

$$
k+l=\left\lfloor\frac{d_{1}}{2}\right\rfloor+\left\lfloor\frac{d_{2}}{2}\right\rfloor-\left\lfloor\frac{d_{3}}{2}\right\rfloor+s_{1}+s_{2}-s_{3}+1 .
$$

Using that $\sum s_{i}$ is odd (2.15), the condition $k+l$ even or odd, translates directly into a condition on $\sum_{i}\left\lfloor\frac{d_{i}}{2}\right\rfloor$. We find thus

$$
\begin{array}{r}
\phi_{d_{1}}\left(z_{1}\right) \phi_{d_{2}}\left(z_{2}\right)=\lim _{\epsilon \rightarrow 0} \lim _{p \rightarrow \infty} n(p)^{2} \frac{1}{2} \sum_{d_{3}, \epsilon}^{\prime} \sum_{\left(r_{3}, s_{3}\right) \in N\left(d_{3}, \epsilon\right)}\left(\frac{\delta_{2}\left(\sum\left\lfloor\frac{d_{i}}{2}\right\rfloor\right)}{\left|z_{12}\right|^{2\left(h_{1}+h_{2}-h_{3}\right)}} D_{d_{1} d_{2}}^{\mathrm{NS}} d_{3} \phi_{r_{3} s_{3}}\left(z_{2}\right)\right. \\
\left.+\frac{\delta_{2}\left(\sum\left\lfloor\frac{d_{i}}{2}\right\rfloor+1\right)}{\left|z_{12}\right|^{2\left(h_{1}+h_{2}-\tilde{h}_{3}\right)}} \tilde{D}_{d_{1} d_{2}}^{\mathrm{NS}} d_{3} \tilde{\phi}_{r_{3} s_{3}}\left(z_{2}\right)\right)+\cdots
\end{array}
$$


The primed sum indicates that we only sum over those $d_{3}$ that satisfy the inequality in (2.15) and the $\epsilon$ indicates that $d_{3}$ is summed over in steps of $\epsilon$. We would like to turn this sum into an integral in the limit $\epsilon \rightarrow 0$ which amounts to replacing the sum by $\frac{1}{\epsilon} \int \mathrm{d} d_{3}$. The sum over the $\phi_{r_{3} s_{3}}$ weighted by the factor $n(p) /|N|$ turns into the field $\phi_{d_{3}}$. So if we choose $n(p)$ such that

$$
n(p)|N| \epsilon^{-1} \rightarrow 1
$$

we obtain a finite OPE in the limit. This amounts to setting

$$
n(p)=\frac{2}{p} \text {. }
$$

We finally obtain

$$
\begin{aligned}
\phi_{d_{1}}\left(z_{1}\right) \phi_{d_{2}}\left(z_{2}\right)=\int_{\mathbb{R}_{+}} & \mathrm{d} d_{3} \frac{1}{2}\left(\frac{1}{\left|z_{12}\right|^{2\left(h_{1}+h_{2}-h_{3}\right)}} P\left(\frac{d_{1}}{4}, \frac{d_{2}}{4}, \frac{d_{3}}{4}\right) D_{d_{1} d_{2}}^{\mathrm{NS}} d_{3} \phi_{d_{3}}\left(z_{2}\right)\right. \\
& \left.+\frac{1}{\left|z_{12}\right|^{2\left(h_{1}+h_{2}-\tilde{h}_{3}\right)}} P\left(\frac{d_{1}+2}{4}, \frac{d_{2}+2}{4}, \frac{d_{3}+2}{4}\right) \tilde{D}_{d_{1} d_{2}}^{\mathrm{NS}} d_{3} \tilde{\phi}_{d_{3}}\left(z_{2}\right)\right)+\cdots
\end{aligned}
$$

Here we restricted the integration domain to the positive numbers using the field identification $d_{3} \rightarrow-d_{3}$. The structure constants $D_{d_{1} d_{2}}^{\mathrm{NS}} d_{3}$ and $\tilde{D}_{d_{1} d_{2}}^{\mathrm{NS}} d_{3}$ in the limit $p \rightarrow \infty$ (given in (2.13) and (2.14) ) are invariant under the replacement $d_{3} \rightarrow-d_{3}$ (which follows from $\Upsilon(x \mid 1)=\Upsilon(2-x \mid 1)$, see appendix B). The function $P$ implements the fusion rules; it is a step function taking the values 0 and 1 , its definition is given in (3.18).

\subsection{Two-point functions}

Let us now discuss the correlation functions. We want a normalisation such that

$$
\left\langle\phi_{d_{1}}\left(z_{1}\right) \phi_{d_{2}}\left(z_{2}\right)\right\rangle=\frac{\delta\left(d_{1}-d_{2}\right)}{\left|z_{12}\right|^{4 h_{1}}} .
$$

To obtain this we have to rescale the correlators, which corresponds to a change of normalisation of the bulk vacuum. Let us denote the scaling factor for the correlation functions by $\gamma^{2}(p)$. Then the two-point correlator in the limiting theory is defined as

$$
\begin{aligned}
\left\langle\phi_{d_{1}}\left(z_{1}\right) \phi_{d_{2}}\left(z_{2}\right)\right\rangle & :=\lim _{\epsilon \rightarrow 0} \lim _{p \rightarrow \infty} \frac{\gamma^{2}(p) n(p)^{2}}{|N|^{2}} \sum_{\left(r_{i} s_{i}\right) \in N\left(d_{i}, \epsilon\right)}\left\langle\phi_{r_{1} s_{1}}\left(z_{1}\right) \phi_{r_{2} s_{2}}\left(z_{2}\right)\right\rangle \\
& =\lim _{\epsilon \rightarrow 0} \lim _{p \rightarrow \infty} \frac{16 \gamma^{2}(p)}{\epsilon^{2} p^{4}} \sum_{\left(r_{1} s_{1}\right) \in N\left(d_{1}, \epsilon\right) \cap N\left(d_{2}, \epsilon\right)} \frac{1}{\left|z_{12}\right|^{4 h_{1}}},
\end{aligned}
$$

where we used that the minimal model fields have normalised two-point functions,

$$
\left\langle\phi_{r_{1} s_{1}}\left(z_{1}\right) \phi_{r_{2} s_{2}}\left(z_{2}\right)\right\rangle=\frac{\delta_{r_{1} r_{2}} \delta_{s_{1} s_{2}}}{\left|z_{12}\right|^{4 h_{1}}} .
$$

(This is valid if both $r_{i}>s_{i} t$, otherwise one has to take field identification into account.) For $\epsilon$ small enough, the number of elements in the intersection of the sets $N\left(d_{i}, \epsilon\right)$ is given by

$$
\left|N\left(d_{1}, \epsilon\right) \cap N\left(d_{2}, \epsilon\right)\right| \sim \frac{p+2}{2}\left(\epsilon-\left|d_{1}-d_{2}\right|\right) \Theta\left(\epsilon-\left|d_{1}-d_{2}\right|\right),
$$


where $\Theta(x)=1$ for $x \geq 0$ and 0 otherwise. The factor of $p / 2$ in (2.24) together with the $16 p^{-4}$ is absorbed by choosing

$$
\gamma^{2}(p)=(p / 2)^{3}
$$

The remaining function $\epsilon^{-2}(\epsilon-|x|) \Theta(\epsilon-|x|)$ converges to the delta distribution $\delta(x)$, so that we obtain the desired normalisation (2.21).

\subsection{Three-point functions}

With the operator product expansion in (2.20) and the normalisation of the two-point function, we can directly write down the three-point correlation functions. For three superconformal primary fields, we find

$$
\begin{aligned}
\left\langle\phi_{d_{1}}\left(z_{1}\right) \phi_{d_{2}}\left(z_{2}\right) \phi_{d_{3}}\left(z_{3}\right)\right\rangle= & \frac{1}{2} P\left(\frac{d_{1}}{4}, \frac{d_{2}}{4}, \frac{d_{3}}{4}\right) D_{d_{1} d_{2}}^{\mathrm{NS}} d_{3}\left|z_{12}\right|^{\left(d_{3}^{2}-d_{1}^{2}-d_{2}^{2}\right) / 4} \\
& \times\left|z_{13}\right|^{\left(d_{2}^{2}-d_{1}^{2}-d_{3}^{2}\right) / 4}\left|z_{23}\right|^{\left(d_{1}^{2}-d_{2}^{2}-d_{3}^{2}\right) / 4} .
\end{aligned}
$$

For two primary fields and one superdescendant field we obtain similarly

$$
\begin{aligned}
\left\langle\phi_{d_{1}}\left(z_{1}\right) \phi_{d_{2}}\left(z_{2}\right) \tilde{\phi}_{d_{3}}\left(z_{3}\right)\right\rangle= & \frac{1}{2} P\left(\frac{d_{1}+2}{4}, \frac{d_{2}+2}{4}, \frac{d_{3}+2}{4}\right) \tilde{D}_{d_{1} d_{2}}^{\mathrm{NS} d_{3}}\left|z_{12}\right|^{\left(d_{3}^{2}+4-d_{1}^{2}-d_{2}^{2}\right) / 4} \\
& \times\left|z_{13}\right|^{\left(d_{2}^{2}-d_{1}^{2}-d_{3}^{2}-4\right) / 4}\left|z_{23}\right|^{\left(d_{1}^{2}-d_{2}^{2}-d_{3}^{2}-4\right) / 4}
\end{aligned}
$$

\subsection{One-point functions on the upper half plane}

Boundary conditions in supersymmetric minimal models that preserve the superconformal symmetry have been analysed in [22] (for $p$ odd). The boundary conditions are parameterised by the Kac labels. For Kac labels $(u, v)$ with $u+v$ even (NS sector), we have two boundary theories $(u, v)_{ \pm}$corresponding to brane and anti-brane. For these boundary conditions the one-point functions for NSNS bulk fields $\phi_{r s}\left(r+s\right.$ even) are given by ${ }^{2}$

$$
\left\langle\phi_{r s}^{\mathrm{NS}}(z)\right\rangle_{(u, v)_{ \pm}}=\frac{1}{|z-\bar{z}|^{2 h_{r s}}} \frac{\sqrt{2}}{\sqrt[4]{p(p+2)}} \frac{\sin \frac{\pi r u}{p} \sin \frac{\pi s v}{p+2}}{\sqrt{\sin \frac{\pi r}{p} \sin \frac{\pi s}{p+2}}},
$$

for RR fields $(r+s$ odd) they read

$$
\left\langle\phi_{r s}^{\mathrm{R}}(z)\right\rangle_{(u, v)_{ \pm}}= \pm \frac{1}{|z-\bar{z}|^{2 h_{r s}}} \frac{2^{\frac{5}{4}}}{\sqrt[4]{p(p+2)}}(-1)^{\frac{u-v}{2}} \frac{\sin \frac{\pi r u}{p} \sin \frac{\pi s v}{p+2}}{\sqrt{\sin \frac{\pi r}{p} \sin \frac{\pi s}{p+2}}} .
$$

The normalisation of the RR fields is chosen such they have the standard two-point function (2.23). There are also boundary theories associated to Ramond labels $(u, v)$ with $u+v$ odd. They correspond to the opposite sign in the gluing condition for the supercurrent at the boundary. We shall not consider those here, but concentrate in the following on the boundary theories $(u, v)_{ \pm}$with $u+v$ even.

When we now discuss boundary conditions in the limit $p \rightarrow \infty$, we essentially have two options. We can take the boundary labels $(u, v)$ fixed, or we can scale them such

\footnotetext{
${ }^{2}$ To distinguish NSNS and RR field we shall use superscripts ${ }^{\mathrm{NS}}$ and ${ }^{\mathrm{R}}$ frequently in this subsection.
} 
that the conformal weight $h_{u v}$ corresponding to the Kac labels stays fixed in the limit. For bosonic minimal models, both options have been investigated leading to one discrete family of boundary conditions [7, 5], and to one continuous family [8]. For the supersymmetric minimal models we shall only consider the case when the boundary labels are taken to be fixed.

To analyse the limit $p \rightarrow \infty$ we should try to rewrite the one-point function for $\phi_{r s}$ such that it depends on $d_{r s}=r-s t$. This is indeed possible due to the identity (for $u+v$ even)

$$
\sin \frac{\pi u r}{p} \sin \frac{\pi v s}{p+2}= \begin{cases}\sin \frac{\pi u d_{r s}}{2 t} \sin \frac{\pi v d_{r s}}{2} & \text { for } r+s \text { even } \\ (-1)^{\frac{u-v}{2}} \sin \left(\frac{\pi u d_{r s}}{2 t}+\frac{\pi u v}{2}\right) \sin \left(\frac{\pi v d_{r s}}{2}+\frac{\pi u v}{2}\right) & \text { for } r+s \text { odd . }\end{cases}
$$

Let us now consider the one-point function for a NSNS bulk field $\phi_{d}^{\mathrm{NS}}$ in the presence of a boundary condition $(u, v)_{ \pm}$. Using the definition (2.10) of $\phi_{d}^{\mathrm{NS}}(z)$ and suppressing the obvious $z$-dependence we obtain

$$
\begin{aligned}
\left\langle\phi_{d}^{\mathrm{NS}}\right\rangle_{(u, v)_{ \pm}} & =\lim _{p \rightarrow \infty} n(p) \gamma(p) \sqrt{\frac{2}{p}} \frac{\sin \frac{\pi u d}{2} \sin \frac{\pi v d}{2}}{\left|\sin \frac{\pi d}{2}\right|} \\
& =\frac{\sin \frac{\pi u d}{2} \sin \frac{\pi v d}{2}}{\left|\sin \frac{\pi d}{2}\right|} .
\end{aligned}
$$

Note that we had to rescale the disc correlator by the factor $\gamma(p)$ (given in (2.25)) that we introduced to obtain correctly normalised sphere correlators. The factor $\gamma(p)$ can be thought of as rescaling the bulk vacuum such that sphere correlators obtain a factor $\gamma^{2}(p)$ while disc correlators are changed by $\gamma(p)$. (This can be understood best from the boundary state formalism, see section 4.2 of [5] for a discussion.)

The one-point functions obtained above are not independent. Because of the identity

$$
\sin \pi u x \sin \pi v x=\sum_{\ell=|u-v|+1,2}^{u+v-1} \sin \pi \ell x \sin \pi x,
$$

where the summation variable $\ell$ is increased in steps of 2 , we can express every one-point function as a sum of one-point functions for boundary labels $(\ell, 1)_{ \pm}$,

$$
\left\langle\phi_{d}^{\mathrm{NS}}\right\rangle_{(u, v)_{ \pm}}=\sum_{\ell=|u-v|+1,2}^{u+v-1}\left\langle\phi_{d}^{\mathrm{NS}}\right\rangle_{(\ell, 1)_{ \pm}} .
$$

This suggests that only the boundary conditions $(\ell, 1)_{ \pm}$are elementary. We have to check that a similar condition is true for the one-point functions of RR fields.

To obtain the one-point functions for RR fields in the limit $p \rightarrow \infty$ we should again rewrite the one-point function (2.29) for $\phi_{r s}^{\mathrm{R}}$ in terms of $d_{r s}$. This is possible due to the identity (2.30). Using the same definition (including the normalisation) of $\phi_{d}^{\mathrm{R}}$ in terms of $\phi_{r s}^{\mathrm{R}}$ as for the NS case (see (2.10)) we find

$$
\left\langle\phi_{d}^{\mathrm{R}}\right\rangle_{(u, v)_{ \pm}}= \pm 2^{\frac{3}{4}} \frac{\sin \frac{\pi}{2}(u d+u v) \sin \frac{\pi}{2}(v d+u v)}{\left|\cos \frac{\pi}{2} d\right|} .
$$


Again we find that the one-point functions are not independent. For $u, v$ odd, the numerator is most easily expressed by cosine-functions, and we have

$$
\cos \pi u x \cos \pi v x=\sum_{\ell=|u-v|+1,2}^{u+v-1}(-1)^{\frac{\ell-|u-v|-1}{2}} \cos \pi \ell x \cos \pi x .
$$

For $u, v$ even we find

$$
\sin \pi u x \sin \pi v x=\sum_{\ell=|u-v|+1,2}^{u+v-1}(-1)^{\frac{\ell-|u-v|-1}{2}} \cos \pi \ell x \cos \pi x .
$$

This shows that the boundary conditions $(u, v)_{ \pm}$really are superpositions of boundary conditions $(\ell, 1)_{ \pm}$, more precisely we have

$$
(u, v)_{+}=(|u-v|+1,1)_{+} \oplus(|u-v|+3,1)_{-} \oplus \cdots \oplus(u+v-1,1)_{(-)} u v .
$$

\section{Limit of super Liouville theory}

\subsection{Preliminaries}

The supersymmetric extension of Liouville theory was first considered in [23]. Shortly after the discovery of the exact three-point function of bosonic Liouville theory in [24, 25], the exact bulk structure constants for the supersymmetric version were found in [16, 17]. The theory depends on a parameter $b$ that determines the central charge,

$$
c=\frac{3}{2}\left(1+2 Q^{2}\right) \quad \text { with } Q=b+\frac{1}{b} .
$$

The primary fields $V_{\alpha}^{\mathrm{NS}}$ in the NSNS sector are labelled by a parameter $\alpha=\frac{Q}{2}+i p$ with real momentum $p$. The conformal weight of $V_{\alpha}^{\mathrm{NS}}$ is given by

$$
h_{\alpha}=\frac{\alpha(Q-\alpha)}{2}=\frac{Q^{2}}{8}+\frac{p^{2}}{2} .
$$

The superdescendant fields $\tilde{V}_{\alpha}^{\mathrm{NS}}$ then have conformal weight $h_{\alpha}+\frac{1}{2}$.

In the Ramond-Ramond sector, the ground state of a representation generically has degeneracy 4 due to the presence of zero modes of the supercurrent. The corresponding RR fields are labelled by $\Theta_{\alpha}^{\epsilon, \bar{\epsilon}}$ with $\epsilon, \bar{\epsilon}= \pm 1$; the OPE with the supercurrent reads

$$
T_{\mathrm{F}}(z) \Theta_{\alpha}^{\epsilon, \bar{\epsilon}}(0) \sim \frac{p \Theta_{\alpha}^{-\epsilon, \bar{\epsilon}}}{\sqrt{2} z^{3 / 2}} \quad-i \Theta_{\alpha}^{\epsilon, \bar{\epsilon}}(0) \bar{T}_{\mathrm{F}}(\bar{z}) \sim \frac{p \Theta_{\alpha}^{\epsilon,-\bar{\epsilon}}}{\sqrt{2} \bar{z}^{3 / 2}} .
$$

Similar to the theory of a free fermion (see e.g. [26]), locality and modular invariance restrict these four fields to one combination $V_{\alpha}^{\mathrm{R}}$, which can be chosen as

$$
V_{\alpha}^{\mathrm{R}}=\frac{1}{\sqrt{2}}\left(\Theta_{\alpha}^{++}+\Theta_{\alpha}^{--}\right)
$$

The conformal weight of $V_{\alpha}^{\mathrm{R}}$ is

$$
h_{\alpha}^{\mathrm{R}}=h_{\alpha}+\frac{1}{16}=\frac{p^{2}}{2}+\frac{c}{24} .
$$




\subsection{Two- and three-point functions: Neveu-Schwarz sector}

The three-point function of primary fields in the Neveu-Schwarz sector reads [18]

$$
\left\langle V_{\alpha_{1}}^{\mathrm{NS}}\left(z_{1}\right) V_{\alpha_{2}}^{\mathrm{NS}}\left(z_{2}\right) V_{\alpha_{3}}^{\mathrm{NS}}\left(z_{3}\right)\right\rangle=C^{\mathrm{NS}}\left(\alpha_{i}\right)\left|z_{12}\right|^{2\left(h_{1}+h_{2}-h_{3}\right)}\left|z_{23}\right|^{2\left(h_{2}+h_{3}-h_{1}\right)}\left|z_{13}\right|^{2\left(h_{1}+h_{3}-h_{2}\right)},
$$

with

$$
C^{\mathrm{NS}}\left(\alpha_{i}\right)=\left(\mu \pi \gamma\left(\frac{b Q}{2}\right) b^{1-b^{2}}\right)^{\frac{Q-2 \tilde{\alpha}}{b}} \frac{\Upsilon_{\mathrm{NS}}^{\prime}(0) \Upsilon_{\mathrm{NS}}\left(2 \alpha_{1}\right) \Upsilon_{\mathrm{NS}}\left(2 \alpha_{2}\right) \Upsilon_{\mathrm{NS}}\left(2 \alpha_{3}\right)}{\Upsilon_{\mathrm{NS}}(2 \tilde{\alpha}-Q) \Upsilon_{\mathrm{NS}}\left(2 \tilde{\alpha}_{1}\right) \Upsilon_{\mathrm{NS}}\left(2 \tilde{\alpha}_{2}\right) \Upsilon_{\mathrm{NS}}\left(2 \tilde{\alpha}_{3}\right)} .
$$

Here, $2 \tilde{\alpha}=\alpha_{1}+\alpha_{2}+\alpha_{3}$ and $\tilde{\alpha}_{i}=\tilde{\alpha}-\alpha_{i}$. The functions $\Upsilon_{\text {NS }}$ are combinations of two $\Upsilon$ functions (see appendix B),

$$
\Upsilon_{\mathrm{NS}}(x)=\Upsilon\left(\frac{x}{2} \mid b\right) \Upsilon\left(\frac{x+Q}{2} \mid b\right) \quad \Upsilon_{\mathrm{R}}=\Upsilon\left(\frac{x+b}{2} \mid b\right) \Upsilon\left(\frac{x+b^{-1}}{2} \mid b\right),
$$

where we also introduced the functions $\Upsilon_{\mathrm{R}}$ that we shall need later. The momenta $\alpha_{i}$ are of the form

$$
\alpha_{i}=\frac{Q}{2}+i p_{i},
$$

with real $p_{i}$. The normalisation is such that the two-point function is given by [18]

$$
\left\langle V_{\alpha_{1}}^{\mathrm{NS}}\left(z_{1}\right) V_{\alpha_{2}}^{\mathrm{NS}}\left(z_{2}\right)\right\rangle=\left|z_{12}\right|^{4 h_{\alpha_{1}}} 2 \pi\left(\delta\left(Q-\alpha_{1}-\alpha_{2}\right)+\delta\left(\alpha_{1}-\alpha_{2}\right) R^{\mathrm{NS}}\left(\alpha_{1}\right)\right),
$$

with the reflection amplitude

$$
R^{\mathrm{NS}}(\alpha)=-\left(\mu \pi \gamma\left(\frac{b Q}{2}\right)\right)^{\frac{Q-2 \alpha}{b}} \frac{\Gamma\left(b\left(\alpha-\frac{Q}{2}\right)\right) \Gamma\left(\frac{1}{b}\left(\alpha-\frac{Q}{2}\right)\right)}{\Gamma\left(-b\left(\alpha-\frac{Q}{2}\right)\right) \Gamma\left(-\frac{1}{b}\left(\alpha-\frac{Q}{2}\right)\right)} .
$$

To get to a theory with central charge $c=\frac{3}{2}$, we have to take the limit $b \rightarrow i$. To perform this limit we need the asymptotics of the functions $\Upsilon$ from [8],

$$
\Upsilon\left(i p+\kappa Q+\mathcal{O}\left(Q^{2}\right)\right)=\frac{1}{2} e^{-\frac{1}{\epsilon}\left(\lambda(p)-\frac{\pi^{2}}{2}\right)} e^{i \pi \frac{p^{2}}{2}} e^{-i \pi(p-2 \kappa+1)\left(p-\lfloor p\rfloor-\frac{1}{2}\right)} \Upsilon(1-p \mid 1)^{-1}\left(1+o\left(\epsilon^{0}\right)\right),
$$

where $\lambda(p)=2 \pi^{2}\left(p-\lfloor p\rfloor-\frac{1}{2}\right)^{2}$, and $\epsilon=2 \pi i Q / b$. (The above asymptotics strictly hold only for non-integer $p$, which will be enough for our purposes, see [8] for further details.) Using this we can determine the asymptotics of the functions $\Upsilon_{N S}$ and $\Upsilon_{R}$,

$$
\begin{aligned}
\Upsilon_{\mathrm{NS}}(Q+2 i p) & \sim \frac{1}{4} e^{-\frac{2}{\epsilon}\left(\lambda(p)-\frac{\pi^{2}}{2}\right)} e^{i \pi p^{2}} e^{i \pi(1-2 p)\left(p-\lfloor p\rfloor-\frac{1}{2}\right)} \Upsilon(1-p \mid 1)^{-2} \\
\Upsilon_{\mathrm{NS}}\left(\frac{Q}{2}+2 i p\right) & \sim \frac{1}{4} e^{-\frac{2}{\epsilon}\left(\lambda(p)-\frac{\pi^{2}}{2}\right)} e^{i \pi p^{2}} e^{-2 \pi i p\left(p-\lfloor p\rfloor-\frac{1}{2}\right)} \Upsilon(1-p \mid 1)^{-2} \\
\Upsilon_{\mathrm{R}}(Q+2 i p) & \sim \frac{1}{4} e^{-\frac{2}{\epsilon}\left(\lambda\left(p+\frac{1}{2}\right)-\frac{\pi^{2}}{2}\right)} e^{i \pi p^{2}+\frac{i \pi}{4}} e^{i \pi(1-2 p)\left(p-\left\lfloor p+\frac{1}{2}\right\rfloor\right)}\left[\Upsilon\left(\frac{1}{2}-p \mid 1\right) \Upsilon\left(\frac{3}{2}-p \mid 1\right)\right]^{-1} \\
\Upsilon_{\mathrm{R}}\left(\frac{Q}{2}+2 i p\right) & \sim \frac{1}{4} e^{-\frac{2}{\epsilon}\left(\lambda\left(p+\frac{1}{2}\right)-\frac{\pi^{2}}{2}\right)} e^{i \pi p^{2}+\frac{i \pi}{4}} e^{-2 \pi i p\left(p-\left\lfloor p+\frac{1}{2}\right\rfloor\right)}\left[\Upsilon\left(\frac{1}{2}-p \mid 1\right) \Upsilon\left(\frac{3}{2}-p \mid 1\right)\right]^{-1}
\end{aligned}
$$


Moreover we need

$$
\Upsilon^{\prime}(0 \mid b)=\frac{2 \pi^{2}}{\epsilon} \Upsilon(1 \mid 1)^{-1}\left(1+o\left(\epsilon^{0}\right)\right)
$$

which leads to

$$
\Upsilon_{\mathrm{NS}}^{\prime}(0)=\frac{\pi^{2}}{2 \epsilon} \Upsilon(1 \mid 1)^{-2}\left(1+o\left(\epsilon^{0}\right)\right)
$$

Putting everything together we can now find the asymptotics of the structure constants,

$$
\begin{aligned}
C^{\mathrm{NS}}\left(\alpha_{i}\right)= & \left(\mu \pi \gamma\left(\frac{b Q}{2}\right)\right)^{\frac{Q-2 \tilde{\alpha}}{b}} \frac{2 \pi^{2}}{\epsilon} e^{-\frac{2}{\epsilon} F\left(\frac{p_{i}}{2}\right)} e^{i \eta\left(p_{i}\right)} \\
& \times\left[\frac{\Upsilon(1-\tilde{p} \mid 1) \Upsilon\left(1-\tilde{p}_{1} \mid 1\right) \Upsilon\left(1-\tilde{p}_{2} \mid 1\right) \Upsilon\left(1-\tilde{p}_{3} \mid 1\right)}{\Upsilon(1 \mid 1) \Upsilon\left(1-p_{1} \mid 1\right) \Upsilon\left(1-p_{2} \mid 1\right) \Upsilon\left(1-p_{3} \mid 1\right)}\right]^{2}\left(1+o\left(\epsilon^{0}\right)\right) .
\end{aligned}
$$

Here, $e^{i \eta\left(p_{i}\right)}$ contains the phase factors from the asymptotics of the functions $\Upsilon_{\mathrm{NS}}$ as well as the limit of $b^{\left(1-b^{2}\right) \frac{Q-2 \tilde{\alpha}}{b}}$, and $F$ is given by

$$
F\left(\frac{p_{i}}{2}\right)=\lambda\left(p_{1}\right)+\lambda\left(p_{2}\right)+\lambda\left(p_{3}\right)-\lambda(\tilde{p})-\lambda\left(\tilde{p}_{1}\right)-\lambda\left(\tilde{p}_{2}\right)-\lambda\left(\tilde{p}_{3}\right)+\frac{\pi^{2}}{2} .
$$

This function is always non-negative, so the limit $P\left(\frac{p_{i}}{2}\right)=\lim _{\epsilon \rightarrow 0} e^{-\frac{2}{\epsilon} F}$ is a step function taking the values 0 and 1 . We find (see appendix $\mathrm{C}$ and [8])

$$
P\left(\frac{p_{i}}{2}\right)=\left\{\begin{array}{l}
1\left\lfloor p_{1}\right\rfloor+\left\lfloor p_{2}\right\rfloor+\left\lfloor p_{3}\right\rfloor \text { even } \\
\quad \text { and }\left|\left\{p_{1}\right\}-\left\{p_{2}\right\}\right| \leq\left\{p_{3}\right\} \leq \min \left(\left\{p_{1}\right\}+\left\{p_{2}\right\}, 2-\left\{p_{1}\right\}-\left\{p_{2}\right\}\right) \\
1\left\lfloor p_{1}\right\rfloor+\left\lfloor p_{2}\right\rfloor+\left\lfloor p_{3}\right\rfloor \text { odd } \\
\quad \text { and }\left|\left\{p_{1}\right\}-\left\{p_{2}\right\}\right| \leq 1-\left\{p_{3}\right\} \leq \min \left(\left\{p_{1}\right\}+\left\{p_{2}\right\}, 2-\left\{p_{1}\right\}-\left\{p_{2}\right\}\right) \\
0 \text { otherwise. }
\end{array}\right.
$$

Now it remains to determine the phase $e^{i \eta\left(p_{i}\right)}$. For the values of the $p_{i}$ such that $F\left(\frac{p_{i}}{2}\right)=0$, the phase simplifies to

$$
e^{i \eta\left(p_{i}\right)}=e^{-i \pi\left(\left\lfloor p_{1}\right\rfloor+\left\lfloor p_{2}\right\rfloor+\left\lfloor p_{3}\right\rfloor\right)+\frac{i \pi}{2}} .
$$

From the expressions (3.18) and (3.19) we see that the three-point function is not analytic in the momenta any more. To compare to the results from the limit of minimal models, we have to rescale the fields by

$$
V_{\alpha}^{\mathrm{NS}} \rightarrow v_{p}^{\mathrm{NS}}=i \frac{\epsilon}{2 \pi}\left(\mu \pi \gamma\left(\frac{b Q}{2}\right)\right)^{\frac{1}{b}\left(\alpha-\frac{Q}{2}\right)} e^{i \pi\lfloor p\rfloor} V_{\alpha}^{\mathrm{NS}},
$$

and the correlators on the sphere by

$$
\langle\cdots\rangle \rightarrow \frac{2 \pi}{\epsilon^{2}}\langle\cdots\rangle
$$

It is easy to see that with these rescalings and identifying $p_{i}=\frac{d_{i}}{2}$, the limit $b \rightarrow i$ reproduces the same two- and three-point function (see (2.21), (2.26)) as the limit of minimal models. 
We now consider correlation functions involving a superdescendant field $\tilde{V}_{\alpha}^{\text {NS }}$. From 18 we find

$$
\left\langle V_{\alpha_{1}}^{\mathrm{NS}}\left(z_{1}\right) V_{\alpha_{2}}^{\mathrm{NS}}\left(z_{2}\right) \tilde{V}_{\alpha_{3}}^{\mathrm{NS}}\left(z_{3}\right)\right\rangle=\tilde{C}^{\mathrm{NS}}\left(\alpha_{i}\right)\left|z_{12}\right|^{2\left(h_{1}+h_{2}-\tilde{h}_{3}\right)}\left|z_{23}\right|^{2\left(h_{2}+\tilde{h}_{3}-h_{1}\right)}\left|z_{13}\right|^{2\left(h_{1}+\tilde{h}_{3}-h_{2}\right)},
$$

with

$$
\tilde{C}^{\mathrm{NS}}\left(\alpha_{i}\right)=i\left(\mu \pi \gamma\left(\frac{b Q}{2}\right) b^{1-b^{2}}\right)^{\frac{Q-2 \tilde{\alpha}}{b}} \frac{2 \Upsilon_{\mathrm{NS}}^{\prime}(0) \Upsilon_{\mathrm{NS}}\left(2 \alpha_{1}\right) \Upsilon_{\mathrm{NS}}\left(2 \alpha_{2}\right) \Upsilon_{\mathrm{NS}}\left(2 \alpha_{3}\right)}{\Upsilon_{\mathrm{R}}(2 \tilde{\alpha}-Q) \Upsilon_{\mathrm{R}}\left(2 \tilde{\alpha}_{1}\right) \Upsilon_{\mathrm{R}}\left(2 \tilde{\alpha}_{2}\right) \Upsilon_{\mathrm{R}}\left(2 \tilde{\alpha}_{3}\right)}
$$

The special functions $\Upsilon_{R}$ have been introduced in (3.8). The superdescendant fields are normalised such that

$$
\left\langle\tilde{V}_{\alpha_{1}}^{\mathrm{NS}}\left(z_{1}\right) \tilde{V}_{\alpha_{2}}^{\mathrm{NS}}\left(z_{2}\right)\right\rangle=-4 h_{\alpha_{1}}^{2}\left|z_{12}\right|^{-2}\left\langle V_{\alpha_{1}}\left(z_{1}\right) V_{\alpha_{2}}\left(z_{2}\right)\right\rangle
$$

To perform the limit $b \rightarrow i$, we use the asymptotics of the functions $\Upsilon_{\mathrm{NS}}$ and $\Upsilon_{\mathrm{R}}$ given in (3.13). Inserting them into the expression for the correlator, we get

$$
\begin{aligned}
\tilde{C}^{\mathrm{NS}}\left(\alpha_{i}\right)= & 2 i\left(\mu \pi \gamma\left(\frac{b Q}{2}\right)\right)^{\frac{Q-2 \tilde{\alpha}}{b}} \frac{2 \pi^{2}}{\epsilon} e^{-\frac{2}{\epsilon} F\left(\frac{p_{i}+1}{2}\right)} e^{i \eta\left(p_{i}+1\right)} \\
& \times \frac{\Upsilon\left(\frac{1}{2}-\tilde{p} \mid 1\right) \Upsilon\left(\frac{3}{2}-\tilde{p} \mid 1\right) \prod_{i=1}^{3} \Upsilon\left(\frac{1}{2}-\tilde{p}_{i} \mid 1\right) \Upsilon\left(\frac{3}{2}-\tilde{p}_{i} \mid 1\right)}{\left[\Upsilon(1 \mid 1) \Upsilon\left(1-p_{1} \mid 1\right) \Upsilon\left(1-p_{2} \mid 1\right) \Upsilon\left(1-p_{3} \mid 1\right)\right]^{2}}\left(1+o\left(\epsilon^{0}\right)\right) .
\end{aligned}
$$

Rescaling

$$
\tilde{V}_{\alpha}^{\mathrm{NS}} \rightarrow \tilde{v}_{p}^{\mathrm{NS}}=-\frac{1}{2 h_{\alpha}} \frac{\epsilon}{2 \pi}\left(\mu \pi \gamma\left(\frac{b Q}{2}\right)\right)^{\frac{1}{b}\left(\alpha-\frac{Q}{2}\right)} e^{i \pi\lfloor p\rfloor} \tilde{V}_{\alpha}^{\mathrm{NS}},
$$

we recover in the limit $b \rightarrow i$ the corresponding three-point correlator (2.27) that was obtained from the limit of minimal models.

\subsection{Two- and three-point functions: Ramond sector}

In the normalisation of [18] the two-point function of two Ramond-Ramond fields is given by

$$
\begin{aligned}
& \left\langle\Theta_{\alpha_{1}}^{ \pm \pm}\left(z_{1}\right) \Theta_{\alpha_{2}}^{ \pm \pm}\left(z_{2}\right)\right\rangle=\left|z_{12}\right|^{-4 h_{\alpha_{1}}-\frac{1}{4}} 2 \pi \delta\left(p_{1}+p_{2}\right) \\
& \left\langle\Theta_{\alpha_{1}}^{ \pm \pm}\left(z_{1}\right) \Theta_{\alpha_{2}}^{\mp \mp}\left(z_{2}\right)\right\rangle=\left|z_{12}\right|^{-4 h_{\alpha_{1}}-\frac{1}{4}} 2 \pi \delta\left(p_{1}-p_{2}\right) \tilde{R}\left(\alpha_{1}\right)
\end{aligned}
$$

with the reflection amplitude

$$
R^{\mathrm{R}}(\alpha)=\left(\mu \pi \gamma\left(\frac{b Q}{2}\right)\right)^{\frac{Q-2 \alpha}{b}} \frac{\Gamma\left(\frac{1}{2}+i p b\right) \Gamma\left(\frac{1}{2}+i p / b\right)}{\Gamma\left(\frac{1}{2}-i p b\right) \Gamma\left(\frac{1}{2}-i p / b\right)} .
$$

For the fields $V_{\alpha}^{\mathrm{R}}$ (see (3.4)) we thus have the two-point function

$$
\left\langle V_{\alpha_{1}}^{\mathrm{R}}\left(z_{1}\right) V_{\alpha_{2}}^{\mathrm{R}}\left(z_{2}\right)\right\rangle=\left|z_{12}\right|^{-4 h_{\alpha_{1}}-\frac{1}{4}} 2 \pi\left(\delta\left(p_{1}+p_{2}\right)+\delta\left(p_{1}-p_{2}\right) R^{\mathrm{R}}\left(\alpha_{1}\right)\right) .
$$


The three-point functions involving RR fields are [18] (omitting the obvious coordinate dependence)

$$
\left\langle V_{\alpha_{1}}^{\mathrm{NS}} \Theta_{\alpha_{2}}^{ \pm \pm} \Theta_{\alpha_{3}}^{\mp \mp}\right\rangle=C^{\mathrm{R}}\left(\alpha_{1}, \alpha_{2}, \alpha_{3}\right) \quad, \quad\left\langle V_{\alpha_{1}}^{\mathrm{NS}} \Theta_{\alpha_{2}}^{ \pm \pm} \Theta_{\alpha_{3}}^{ \pm \pm}\right\rangle=\tilde{C}^{\mathrm{R}}\left(\alpha_{1}, \alpha_{2}, \alpha_{3}\right),
$$

with

$$
\begin{aligned}
& C^{\mathrm{R}}\left(\alpha_{i}\right)=\left(\mu \pi \gamma\left(\frac{b Q}{2}\right) b^{1-b^{2}}\right)^{\frac{Q-2 \tilde{\alpha}}{b}} \frac{\Upsilon_{\mathrm{NS}}^{\prime}(0) \Upsilon_{\mathrm{NS}}\left(2 \alpha_{1}\right) \Upsilon_{\mathrm{R}}\left(2 \alpha_{2}\right) \Upsilon_{\mathrm{R}}\left(2 \alpha_{3}\right)}{\Upsilon_{\mathrm{R}}(2 \tilde{\alpha}-Q) \Upsilon_{\mathrm{R}}\left(2 \tilde{\alpha}_{1}\right) \Upsilon_{\mathrm{NS}}\left(2 \tilde{\alpha}_{2}\right) \Upsilon_{\mathrm{NS}}\left(2 \tilde{\alpha}_{3}\right)} \\
& \tilde{C}^{\mathrm{R}}\left(\alpha_{i}\right)=\left(\mu \pi \gamma\left(\frac{b Q}{2}\right) b^{1-b^{2}}\right)^{\frac{Q-2 \tilde{\alpha}}{b}} \frac{\Upsilon_{\mathrm{NS}}^{\prime}(0) \Upsilon_{\mathrm{NS}}\left(2 \alpha_{1}\right) \Upsilon_{\mathrm{R}}\left(2 \alpha_{2}\right) \Upsilon_{\mathrm{R}}\left(2 \alpha_{3}\right)}{\Upsilon_{\mathrm{NS}}(2 \tilde{\alpha}-Q) \Upsilon_{\mathrm{NS}}\left(2 \tilde{\alpha}_{1}\right) \Upsilon_{\mathrm{R}}\left(2 \tilde{\alpha}_{2}\right) \Upsilon_{\mathrm{R}}\left(2 \tilde{\alpha}_{3}\right)}
\end{aligned}
$$

The three-point function involving the fields $V_{\alpha}^{\mathrm{R}}$ are then given by

$$
\left\langle V_{\alpha_{1}}^{\mathrm{NS}} V_{\alpha_{2}}^{\mathrm{R}} V_{\alpha_{3}}^{\mathrm{R}}\right\rangle=C^{\mathrm{R}}\left(\alpha_{i}\right)+\tilde{C}^{\mathrm{R}}\left(\alpha_{i}\right) .
$$

Let us now analyse the limit $b \rightarrow i$. The necessary formulae for the asymptotic behaviour of $\Upsilon_{\mathrm{NS}}$ and $\Upsilon_{\mathrm{R}}$ are given in (3.13). In the limit, the structure constants $C^{\mathrm{R}}$ and $\tilde{C}^{\mathrm{R}}$ behave as

$$
\begin{aligned}
C^{\mathrm{R}}\left(\alpha_{i}\right) \sim & \left(\mu \pi \gamma\left(\frac{b Q}{2}\right)\right)^{\frac{Q-2 \tilde{\alpha}}{b}} \frac{2 \pi^{2}}{\epsilon} e^{-\frac{2}{\epsilon} F\left(\frac{p_{1}}{2}, \frac{p_{2}+\frac{1}{2}}{2}, \frac{p_{3}+\frac{1}{2}}{2}\right)} e^{i \eta\left(p_{1}, p_{2}+\frac{1}{2}, p_{3}+\frac{1}{2}\right)+i \pi} \\
& \times \frac{\Upsilon\left(\frac{1}{2}-\tilde{p} \mid 1\right) \Upsilon\left(\frac{3}{2}-\tilde{p} \mid 1\right) \Upsilon\left(\frac{1}{2}-\tilde{p}_{1} \mid 1\right) \Upsilon\left(\frac{3}{2}-\tilde{p}_{1} \mid 1\right) \Upsilon\left(1-\tilde{p}_{2} \mid 1\right)^{2} \Upsilon\left(1-\tilde{p}_{3} \mid 1\right)^{2}}{\Upsilon(1 \mid 1)^{2} \Upsilon\left(1-p_{1} \mid 1\right)^{2} \Upsilon\left(\frac{1}{2}-p_{2} \mid 1\right) \Upsilon\left(\frac{3}{2}-p_{2} \mid 1\right) \Upsilon\left(\frac{1}{2}-p_{3} \mid 1\right) \Upsilon\left(\frac{3}{2}-p_{3} \mid 1\right)} \\
\tilde{C}^{\mathrm{R}}\left(\alpha_{i}\right) \sim & \left(\mu \pi \gamma\left(\frac{b Q}{2}\right)\right)^{\frac{Q-2 \tilde{\alpha}}{2}} \frac{2 \pi^{2}}{\epsilon} e^{-\frac{2}{\epsilon} F\left(\frac{p_{1}}{2}, \frac{p_{2}-\frac{1}{2}}{2}, \frac{p_{3}+\frac{1}{2}}{2}\right)} e^{i \eta\left(p_{1}, p_{2}-\frac{1}{2}, p_{3}+\frac{1}{2}\right)} \\
& \times \frac{\Upsilon(1-\tilde{p} \mid 1)^{2} \Upsilon\left(1-\tilde{p}_{1} \mid 1\right)^{2} \Upsilon\left(\frac{1}{2}-\tilde{p}_{2} \mid 1\right) \Upsilon\left(\frac{3}{2}-\tilde{p}_{2} \mid 1\right) \Upsilon\left(\frac{1}{2}-\tilde{p}_{3} \mid 1\right) \Upsilon\left(\frac{3}{2}-\tilde{p}_{3} \mid 1\right)}{\Upsilon(1 \mid 1)^{2} \Upsilon\left(1-p_{1} \mid 1\right)^{2} \Upsilon\left(\frac{1}{2}-p_{2} \mid 1\right) \Upsilon\left(\frac{3}{2}-p_{2} \mid 1\right) \Upsilon\left(\frac{1}{2}-p_{3} \mid 1\right) \Upsilon\left(\frac{3}{2}-p_{3} \mid 1\right)}
\end{aligned}
$$

Again we have to rescale the fields to obtain a finite limit. Choosing the rescaling

$$
\Theta_{\alpha}^{ \pm \pm} \rightarrow \theta_{p}^{ \pm \pm}=\frac{\epsilon}{2 \pi}\left(\mu \pi \gamma\left(\frac{b Q}{2}\right)\right)^{i p / b} e^{i \pi\left\lfloor p-\frac{1}{2}\right\rfloor} \Theta_{\alpha}^{ \pm \pm},
$$

and similarly $V_{\alpha}^{\mathrm{R}} \rightarrow v_{p}^{\mathrm{R}}$, we obtain for $b \rightarrow i$ the normalised two-point function

$$
\left\langle v_{p_{1}}^{\mathrm{R}}\left(z_{1}\right) v_{p_{2}}^{\mathrm{R}}\left(z_{2}\right)\right\rangle=\left|z_{12}\right|^{-2 p_{1}^{2}-\frac{1}{4}}\left(\delta\left(p_{1}+p_{2}\right)+\delta\left(p_{1}-p_{2}\right)\right) .
$$

The limit of the three-point functions is then given by

$$
\begin{aligned}
\left\langle v_{p_{1}}^{\mathrm{NS}} \theta_{p_{2}}^{ \pm \pm} \theta_{p_{3}}^{\mp \mp}\right\rangle= & \frac{1}{2} P\left(\frac{p_{1}}{2}, \frac{p_{2}+\frac{1}{2}}{2}, \frac{p_{3}+\frac{1}{2}}{2}\right) \\
& \times \frac{\Upsilon\left(\frac{1}{2}-\tilde{p} \mid 1\right) \Upsilon\left(\frac{3}{2}-\tilde{p} \mid 1\right) \Upsilon\left(\frac{1}{2}-\tilde{p}_{1} \mid 1\right) \Upsilon\left(\frac{3}{2}-\tilde{p}_{1} \mid 1\right) \Upsilon\left(1-\tilde{p}_{2} \mid 1\right)^{2} \Upsilon\left(1-\tilde{p}_{3} \mid 1\right)^{2}}{\Upsilon(1 \mid 1)^{2} \Upsilon\left(1-p_{1} \mid 1\right)^{2} \Upsilon\left(\frac{1}{2}-p_{2} \mid 1\right) \Upsilon\left(\frac{3}{2}-p_{2} \mid 1\right) \Upsilon\left(\frac{1}{2}-p_{3} \mid 1\right) \Upsilon\left(\frac{3}{2}-p_{3} \mid 1\right)} \\
\left\langle v_{p_{1}}^{\mathrm{NS}} \theta_{p_{2}}^{ \pm \pm} \theta_{p_{3}}^{ \pm \pm}\right\rangle= & \frac{1}{2} P\left(\frac{p_{1}}{2}, \frac{p_{2}-\frac{1}{2}}{2}, \frac{p_{3}+\frac{1}{2}}{2}\right) \\
& \times \frac{\Upsilon(1-\tilde{p} \mid 1)^{2} \Upsilon\left(1-\tilde{p}_{1} \mid 1\right)^{2} \Upsilon\left(\frac{1}{2}-\tilde{p}_{2} \mid 1\right) \Upsilon\left(\frac{3}{2}-\tilde{p}_{2} \mid 1\right) \Upsilon\left(\frac{1}{2}-\tilde{p}_{3} \mid 1\right) \Upsilon\left(\frac{3}{2}-\tilde{p}_{3} \mid 1\right)}{\Upsilon(1 \mid 1)^{2} \Upsilon\left(1-p_{1} \mid 1\right)^{2} \Upsilon\left(\frac{1}{2}-p_{2} \mid 1\right) \Upsilon\left(\frac{3}{2}-p_{2} \mid 1\right) \Upsilon\left(\frac{1}{2}-p_{3} \mid 1\right) \Upsilon\left(\frac{3}{2}-p_{3} \mid 1\right)}
\end{aligned}
$$

Note that the correlators (3.39) and (3.40) transform into each other when $p_{3} \rightarrow-p_{3}$. 


\subsection{One-point functions on the upper half plane}

Boundary conditions for supersymmetric Liouville theory have been analysed in [27, 18]. There is a discrete family (the analogue of the ZZ-branes [9] in bosonic Liouville theory) and a continuous family (corresponding to the FZZT-branes [10, 11]). We shall concentrate on the discrete family here and try to reproduce the results that we obtained from the minimal models. These boundary conditions are labelled by two integers $(u, v)$ where the sign of the gluing condition for the supercurrent depends on $u+v$ being even or odd. Similarly to the analysis for the minimal models we focus on the case $u+v$ even. Then there are two boundary conditions $(u, v)_{ \pm}$for each tuple, differing in the sign of one-point functions for RR-fields.

The one-point functions (omitting the $z$-dependence) are 18

$$
\left\langle V_{\alpha}^{\mathrm{NS}}\right\rangle_{(u, v)_{ \pm}}=-2 \sqrt{\frac{2}{\pi}}\left(\mu \pi \gamma\left(\frac{b Q}{2}\right)\right)^{-i p / b} i p \Gamma(i p b) \Gamma(i p / b) \sinh \pi p u b \sinh \pi p v / b
$$

and

$$
\begin{aligned}
\left\langle V_{\alpha}^{\mathrm{R}}\right\rangle_{(u, v)_{ \pm}}= & \pm 2^{3 / 4} \sqrt{\frac{2}{\pi}}\left(\mu \pi \gamma\left(\frac{b Q}{2}\right)\right)^{-\frac{i p}{b}} \Gamma\left(\frac{1}{2}+i p b\right) \Gamma\left(\frac{1}{2}+\frac{i p}{b}\right) \times \\
& \times \sinh \left(\pi p u b+\frac{i \pi u v}{2}\right) \sinh \left(\frac{\pi p v}{b}-\frac{i \pi u v}{2}\right)
\end{aligned}
$$

When we take the limit $b \rightarrow i$ we have to rescale the sphere correlators by the factor $\frac{2 \pi}{\epsilon^{2}}$ (see (3.21)). According to the discussion in section 2.6 the scaling factor for the disc correlators should be the square-root of it. To match the results of the minimal models we have to choose the root with negative sign, so

$$
\langle\cdots\rangle_{(u, v)_{ \pm}} \rightarrow-\frac{\sqrt{2 \pi}}{\epsilon}\langle\cdots\rangle_{(u, v)_{ \pm}}
$$

By going to the rescaled NSNS bulk fields $v_{p}^{\mathrm{NS}}(3.20)$ we find

$$
\begin{aligned}
\left\langle v_{p}^{\mathrm{NS}}\right\rangle_{(u, v)_{ \pm}} & =\frac{1}{\pi} e^{i \pi\lfloor p\rfloor} \Gamma(1-p) \Gamma(p) \sin \pi p u \sin \pi p v \\
& =\frac{\sin \pi p u \sin \pi p v}{|\sin \pi p|}
\end{aligned}
$$

which coincides with the expression (2.31) we found from the minimal models. For the RR fields we obtain similarly

$$
\left\langle v_{p}^{\mathrm{R}}\right\rangle_{(u, v)_{ \pm}}= \pm 2^{\frac{3}{4}} \frac{\sin \left(\pi p u+\frac{\pi u v}{2}\right) \sin \left(\pi p v+\frac{\pi u v}{2}\right)}{|\cos \pi p|}
$$

and we find again complete agreement with the minimal model result (2.34). 


\section{Summary and outlook}

In this paper we have analysed the limit of supersymmetric minimal models at central charge $c=\frac{3}{2}$. We computed the three-point correlation functions for the limiting theory and showed that it is possible to get the same correlators from a limit of supersymmetric Liouville theory. In addition we obtained the one-point functions in the presence of a boundary for a discrete family of boundary conditions.

The computation of the three-point correlators cannot be seen as a rigorous construction of the theory. The two ways of taking limits both are singular: the transition from a discrete to a continuous spectrum in the minimal model limit, and the loss of analyticity in the limit of the Liouville correlators. Although the fact that we can obtain the same correlators on two different paths makes it plausible that the resulting theory is a healthy conformal field theory, this is not guaranteed. Further checks on the theory should be performed, in particular one should analyse whether the theory is crossing symmetric. For the bosonic counterpart of the theory, this has been analysed in [5], on the one hand analytically for specific four-point correlators, and on the other hand numerically for the generic case. Similar checks could also be performed in the supersymmetric case, numerical checks could use the recently found recursion relations for superconformal blocks [28, 29]. Instead of directly testing the crossing symmetry, one would expect that it can be derived from the crossing symmetry valid for the Liouville four-point correlators.

In this work we concentrated mostly on the Neveu-Schwarz sector of the theory. In super Liouville theory, three-point functions involving Ramond-Ramond fields have been determined and we used this result to obtain their limit at $c=\frac{3}{2}$. On the other hand, in the case of super minimal models the corresponding structure constants have not been determined explicitly (see however [30, 31] for some results), so before comparing the two limits it would be necessary to compute theses quantities first.

We discussed a discrete family of boundary conditions and computed the bulk onepoint function. In addition there is another discrete family (to which the RR fields do not couple) and also a continuous family. It should be straightforward to determine the limits of one-point functions for those. Further data, like bulk-boundary operator expansion and boundary operator product expansion, however, will be much more difficult to determine (for the bosonic case, the limit of the bulk-boundary operator expansion for the continuous family of branes was determined in [8], the limit of the boundary OPE is still unknown).

One might now try to perform a similar analysis for the $N=2$ minimal models and the $N=2$ Liouville theory. The $N=2$ minimal models can be built as supersymmetric cosets $s u(2) / u(1)$, and their structure constants are known [32-34]. They differ significantly from the bosonic or $N=1$ case, but it is still possible to find analytic expressions that interpolate the three-point functions to continuous $s u(2)$ spins [35], and it is conceivable that there exist a non-trivial limit at $c=3 .{ }^{3}$ On the other hand, also the Liouville structure constants are known in the $N=2$ case [37], by a coset construction [38] they are related to those of the $H_{3}^{+}$model [39]. They are again built from Barnes' double gamma function,

\footnotetext{
${ }^{3}$ Aspects of the asymptotic behaviour of the $s u(2)$ structure constants have been analysed in a different context in 36].
} 
but the limit that leads to central charge $c=3$ now corresponds to taking $b \rightarrow 0$ instead of $b \rightarrow i$. Again, a non-trivial limit might exist, but the asymptotics will be completely different from the bosonic or $N=1$ case.

The limit of Virasoro minimal models can be viewed as a limit of $s u(2)$ coset models; analogously it should be possible to take such a limit for all diagonal coset models of the type

$$
\frac{\mathfrak{g}_{k} \times \mathfrak{g}_{1}}{\mathfrak{g}_{k+1}}
$$

with some simply laced Lie algebra $\mathfrak{g}$. The limiting central charge is $c=c\left(\mathfrak{g}_{1}\right)=\operatorname{rank} \mathfrak{g}$. On the other hand, one can study the Toda conformal field theory with Lie algebra $\mathfrak{g}$ whose central charge approaches the same value $c=\operatorname{rank} \mathfrak{g}$ in the limit $b \rightarrow i$. One might suspect that the limits again lead to the same conformal field theory, but as only a few explicit results on the structure constants are available (see e.g. [40] for the conformal Toda theories and [41] for the minimal models), we leave this analysis for future research.

When we continue the expressions from bosonic Liouville theory to $c=1$, one might wonder whether it is also possible to continue them to $c<1$. This is in fact straightforward for rational central charges of the form $c=1-6 \frac{(p-q)^{2}}{p q}$. The corresponding conformal field theories would not be unitary and would have a continuous spectrum. Again the limit of the structure constants is the product of an analytic part which coincides with the analytic interpolation of the minimal model structure constants and a step function. Recently this limit was investigated in [42], and it was argued that only for $p=1$ a physically sensible three-point function is obtained. The relation of the resulting theory to minimal models or generalised minimal models 43, 44 is unclear until now. The continuation of Liouville theory to other, non-rational central charges $c<1$ is more singular, and it is clear that the structure constants do not have a well-defined limit as functions of the field parameters, though it might be that one can find a sensible limit in terms of distributions.

\section{Acknowledgments}

We are grateful to Giuseppe D'Appollonio, Matthias Gaberdiel, Ingo Runkel and Stefan Theisen for useful discussions. This paper is in part based on the diploma thesis of one of the authors 45. SF acknowledges support from the Swiss National Science Foundation (SNF) for his time at ETH Zürich where the project was started.

\section{A. Structure constants in terms of Barnes' double gamma functions}

\section{A.1 Virasoro minimal models}

The unitary minimal models are labelled by an integer $p$; the central charge is given by $c=1-\frac{6}{p(p+1)}$. The set of primary fields $\phi_{r s}$ is specified by the Kac table, which consists of pairs of integers $(r, s)$ with $1 \leq r \leq p-1$ and $1 \leq s \leq p$. The conformal weight of a primary field $\phi_{r s}$ is given by

$$
h_{r s}=\frac{((p+1) r-p s)^{2}-1}{4 p(p+1)}
$$


The pairs $(r, s)$ and $(p-r, p+1-s)$ label the same field.

The operator product expansion for two primary fields is given by

$$
\phi_{r_{1} s_{1}}\left(z_{1}\right) \phi_{r_{2} s_{2}}\left(z_{2}\right) \sim \sum_{r_{3}, s_{3}} \mathcal{N}_{r_{1} r_{2}}^{(p) r_{3}} \mathcal{N}_{s_{1} s_{2}}^{(p+1) s_{3}} \frac{D_{\left(r_{1} s_{1}\right)\left(r_{2} s_{2}\right)}^{\left(r_{3} s_{3}\right)}}{\left|z_{12}\right|^{2\left(h_{r_{1} s_{1}}+h_{r_{2} s_{2}}-h_{\left.r_{3} s_{3}\right)}\right.}} \phi_{r_{3} s_{3}}\left(z_{2}\right) .
$$

Here, $\mathcal{N}^{(p)} \mathcal{N}^{(p+1)}$ implements the fusion constraints, and $\mathcal{N}^{(p)}$ is defined as

$$
\mathcal{N}_{r_{1} r_{2}}^{(p)} r_{3}=\left\{\begin{array}{l}
1\left|r_{1}-r_{2}\right|+1 \leq r_{3} \leq \min \left(r_{1}+r_{2}-1,2 p-r_{1}-r_{2}-1\right), \quad \sum_{i} r_{i} \text { odd } \\
0 \text { otherwise }
\end{array}\right.
$$

The structure constants $D$ have been determined originally by Dotsenko and Fateev 46 48]. We want them to be normalised such that $D_{(r s)(r s)}{ }^{(11)}=1$. It is convenient to express them by structure constants $C$ in a different normalisation of the fields,

$$
\begin{aligned}
C_{\left(r_{1} s_{1}\right)\left(r_{2} s_{2}\right)}^{\left(r_{3} s_{3}\right)}= & t^{4(k-1)(l-1)} \prod_{m=1}^{k-1} \prod_{n=1}^{l-1}\left(d_{m n}\left(d_{m n}-d_{1}\right)\left(d_{m n}-d_{2}\right)\left(d_{m n}+d_{3}\right)\right)^{-2} \\
& \times \prod_{m=1}^{k-1} \frac{\Gamma\left(\frac{m}{t}\right) \Gamma\left(\frac{m-d_{1}}{t}\right) \Gamma\left(\frac{m-d_{2}}{t}\right) \Gamma\left(\frac{m+d_{3}}{t}\right)}{\Gamma\left(1-\frac{m}{t}\right) \Gamma\left(1-\frac{m-d_{1}}{t}\right) \Gamma\left(1-\frac{m-d_{2}}{t} \Gamma\left(1-\frac{m+d_{3}}{t}\right)\right)} \\
& \times \prod_{n=1}^{l-1} \frac{\Gamma(n t) \Gamma\left(n t+d_{1}\right) \Gamma\left(n t+d_{2}\right) \Gamma\left(n t-d_{3}\right)}{\Gamma(1-n t) \Gamma\left(1-n t-d_{1}\right) \Gamma\left(1-n t-d_{2}\right) \Gamma\left(1-n t+d_{3}\right)} .
\end{aligned}
$$

Here $k=\frac{r_{1}+r_{2}-r_{3}+1}{2}, l=\frac{s_{1}+s_{2}-s_{3}+1}{2}, d_{m n}=m-n t, d_{i}=d_{r_{i} s_{i}}, t=\frac{p}{p+1}$, and the normalised structure constants can be recovered as

$$
D_{\left(r_{1} s_{1}\right)\left(r_{2} s_{2}\right)}^{\left(r_{3} s_{3}\right)}=\sqrt{\frac{C_{\left(r_{3} s_{3}\right)\left(r_{3} s_{3}\right)}^{(11)}}{C_{\left(r_{1} s_{1}\right)\left(r_{1} s_{1}\right)}{ }^{(11)} C_{\left(r_{2} s_{2}\right)\left(r_{2} s_{2}\right)}^{(11)}}} C_{\left(r_{1} s_{1}\right)\left(r_{2} s_{2}\right)}{ }^{\left(r_{3} s_{3}\right)} .
$$

The expression for the structure constants is also valid for the non-unitary minimal models where $t=\frac{p}{q}$ is any rational number. All results that will be obtained in this appendix will only involve this parameter $t$, and therefore apply to all minimal models.

Dotsenko made the observation that the structure constants only depend on a linear combination $d_{r_{i} s_{i}}$ of the Kac labels $\left(r_{i}, s_{i}\right)$, and that they are analytic in the variables $d_{r_{i} s_{i}}$. From the expression (A.4) this is not obvious because the Kac labels enter the range of products via $k$ and $l$. Although the result seems to be well known, there is, to our knowledge, no explicit proof of this statement in the literature. We therefore felt it could be useful to give a detailed derivation of this fact here. In the following we shall re-express the structure constants in terms of special functions, such that its analyticity in $d_{r_{i} s_{i}}$ is manifest. The expression we derive has been given recently in [43] for the three-point function in generalised minimal models without explicitly showing that it reproduces the Dotsenko-Fateev structure constants.

Let us briefly describe the general strategy. Suppose you are given a finite product of functions with equally spaced arguments, $\prod_{m=1}^{k-1} f(m)$, and you would like to obtain an expression that is analytic in the range $k$ of the product and that interpolates between 
integer values of $k$. If one can find an analytic function $F$ with the shift property $F(x+1)=$ $f(x) F(x)$, one can rewrite the product as a quotient,

$$
\prod_{m=1}^{k-1} f(m)=\frac{F(k)}{F(1)}
$$

which provides a manifestly analytic expression in $k$. This expression is of course not unique and depends on the choice of $F$.

The first product in the expression (A.4) of the structure constants is a double product in the variables $m, n$ over numbers depending linearly on $m$ and $n$. A product over equally spaced numbers can be replaced by a ratio of gamma functions in two ways,

$$
\prod_{m=1}^{k-1}(m+x)=\frac{\Gamma(x+k)}{\Gamma(x+1)}=(-1)^{k-1} \frac{\Gamma(-x)}{\Gamma(-x-k+1)} .
$$

By this means we can transform the double product into a single product of quotients of gamma functions. The numbers in the double product come as squares, so we can use both ways in (A.7) to rewrite the double product as

$$
\prod_{m=1}^{k-1} \prod_{n=1}^{l-1}(x+m-n t)^{-2}=(-1)^{(k-1)(l-1)} \prod_{n=1}^{l-1} \frac{\Gamma(x-n t+1)}{\Gamma(x-n t+k)} \frac{\Gamma(-x+n t-k+1)}{\Gamma(-x+n t)},
$$

where $x$ stands for the possible values $x=0,-d_{1},-d_{2}, d_{3}$. Using this result we can write the structure constants $C$ as

$$
C_{\left(r_{1} s_{1}\right)\left(r_{2} s_{2}\right)}^{\left(r_{3} s_{3}\right)}=t^{4(k-1)(l-1)} \cdot \prod_{x=0,-d_{1},-d_{2}, d_{3}}\left(\prod_{m=1}^{k-1} \frac{\Gamma\left(\frac{x+m}{t}\right)}{\Gamma\left(1-\frac{x+m}{t}\right)} \prod_{n=1}^{l-1} \frac{\Gamma(-x+n t-k+1)}{\Gamma(x-n t+k)}\right) .
$$

Now we only have to deal with products of gamma functions. The crucial step in rewriting the structure constants consists in realising that a product over gamma functions with equally spaced entries can be expressed as a quotient of Barnes' double gamma functions,

$$
\prod_{m=1}^{k-1} \Gamma\left(\frac{m+x}{t}\right)=(2 \pi)^{\frac{k-1}{2}} t^{-\frac{k-1}{2 t}\left(x+\frac{k}{2}-\frac{t}{2}\right)} \frac{\Gamma_{2}\left((1+x) t^{-\frac{1}{2}} \mid t^{-\frac{1}{2}}\right)}{\Gamma_{2}\left((k+x) t^{-\frac{1}{2}} \mid t^{-\frac{1}{2}}\right)} .
$$

This can be easily verified using the shift property (B.2) of the double gamma function $\Gamma_{2}$.

With the help of identity (A.10) we replace the first product of gamma functions in (A.4) and obtain

$$
\prod_{m=1}^{k-1} \frac{\Gamma\left(\frac{m+x}{t}\right)}{\Gamma\left(1-\frac{m+x}{t}\right)}=t^{-\frac{k-1}{t}\left(x+\frac{k}{2}-\frac{t}{2}\right)} \frac{\Upsilon\left((x+k) t^{-\frac{1}{2}} \mid t^{-\frac{1}{2}}\right)}{\Upsilon\left((x+1) t^{-\frac{1}{2}} \mid t^{-\frac{1}{2}}\right)},
$$

where the function $\Upsilon$ is defined by (see $(\overline{B .4}))$

$$
\Upsilon\left(y \mid t^{-\frac{1}{2}}\right)=\left[\Gamma_{2}\left(y \mid t^{-\frac{1}{2}}\right) \Gamma_{2}\left(t^{\frac{1}{2}}+t^{-\frac{1}{2}}-y \mid t^{-\frac{1}{2}}\right)\right]^{-1} .
$$


We can apply the same procedure to the second product in (A.9), bearing in mind that $\Gamma_{2}\left(x \mid t^{-\frac{1}{2}}\right)=\Gamma_{2}\left(x \mid t^{\frac{1}{2}}\right)$. We find

$$
\prod_{n=1}^{l-1} \frac{\Gamma(-x+n t-k+1)}{\Gamma(x-n t+k)}=t^{-(l-1)\left(x+k-\frac{1}{2}-\frac{l t}{2}\right)} \frac{\Upsilon\left((-x+l t-k+1) t^{-\frac{1}{2}} \mid t^{-\frac{1}{2}}\right)}{\Upsilon\left((x+k) t^{-\frac{1}{2}} \mid t^{-\frac{1}{2}}\right)} .
$$

Putting the results together we get

$$
C_{\left(r_{1} s_{1}\right)\left(r_{2} s_{2}\right)}^{\left(r_{3} s_{3}\right)}=t^{-(1-t)\left(\frac{k-1}{t}+(l-1)\right)} \cdot \prod_{x=0,-d_{1},-d_{2}, d_{3}} \frac{\Upsilon\left(\left(-x-d_{k l}+1\right) t^{-\frac{1}{2}} \mid t^{-\frac{1}{2}}\right)}{\Upsilon\left((x+1) t^{-\frac{1}{2}} \mid t^{-\frac{1}{2}}\right)} .
$$

Except for the exponent of $t$ in the factor in front, the structure constant is expressed entirely in the variables $d_{i}$ (note that $d_{k l}=\frac{d_{1}+d_{2}-d_{3}+1-t}{2}$ ). The $(r, s)$-dependent part of the power of $t$ will drop out in the normalised structure constants $D$. To determine them via A.5 ) we first observe that

$$
C_{(r s)(r s)}^{(11)}=t^{-(1-t)\left(\frac{r-1}{t}+(s-1)\right)} \frac{\Upsilon\left(\left(d_{r s}+1\right) t^{-\frac{1}{2}} \mid t^{-\frac{1}{2}}\right) \Upsilon\left(t^{-\frac{1}{2}} \mid t^{-\frac{1}{2}}\right)}{\Upsilon\left(\left(d_{r s}+t\right) t^{-\frac{1}{2}} \mid t^{-\frac{1}{2}}\right) \Upsilon\left((2-t) t^{-\frac{1}{2}} \mid t^{-\frac{1}{2}}\right)} .
$$

The normalised structure constants $D$ then only depend on the variables $d_{i}$ and we obtain

$$
D_{\left(r_{1} s_{1}\right)\left(r_{2} s_{2}\right)}^{\left(r_{3} s_{3}\right)}=D_{0} \prod_{x=0,-d_{1},-d_{2}, d_{3}} \frac{\Upsilon\left(\left(-x-d_{k l}+1\right) t^{-\frac{1}{2}} \mid t^{-\frac{1}{2}}\right)}{\left[\Upsilon\left((x+1) t^{-\frac{1}{2}} \mid t^{-\frac{1}{2}}\right) \Upsilon\left((x+t) t^{-\frac{1}{2}} \mid t^{-\frac{1}{2}}\right)\right]^{\frac{1}{2}}},
$$

with the normalisation constant

$$
D_{0}=t^{-\frac{t-t^{-1}-1}{2}}\left[\gamma\left(1-t^{-1}\right) \gamma(2-t)\right]^{-\frac{1}{2}} .
$$

Note that our derivation is also valid for the non-unitary minimal models where $t=\frac{p}{q}$ is any rational number. The expression (A.16) coincides with the formula given in [43]. The normalised structure constants $D$ are invariant under permutation of the variables $d_{i}$. Furthermore, they do not change if one performs field identifications in two field labels (e.g. $d_{1} \rightarrow-d_{1}$ and $d_{2} \rightarrow-d_{2}$ ) at the same time.

Let us discuss the uniqueness of our result. For a fixed minimal model there are only a finite number of fields, and the analytic interpolation of the structure constants is of course not unique. As already stated above, the Dotsenko-Fateev expressions apply to any rational $t$. If we require the interpolation to be continuous in the parameter $t$, then it is uniquely fixed: any given $d$ and $t$ can be obtained as a limit of rational $d_{r_{i} s_{i}}$ and $t_{i}$.

\section{A.2 Superconformal minimal models}

The $N=1$ supersymmetric minimal models are labelled by an integer $p$, and they have central charge

$$
c=\frac{3}{2}\left(1-\frac{8}{p(p+2)}\right)
$$


The primary fields $\phi_{r s}$ are labelled by pairs of integers $(r, s)$ in the range $1 \leq r \leq p-1$ and $1 \leq s \leq p+1$. Two pairs $(r, s)$ and $(p-r, p+2-s)$ label the same field. Depending on the sum $r+s$ being even or odd, the corresponding primary field belongs to the Neveu-Schwarz or Ramond sector, respectively.

We shall only consider the Neveu-Schwarz sector. The operator product expansion of two primary fields is given by

$$
\begin{aligned}
\phi_{r_{1} s_{1}}\left(z_{1}\right) \phi_{r_{2} s_{2}}\left(z_{2}\right) \sim & \sum_{r_{3}, s_{3}} \mathcal{N}_{r_{1} r_{2}}^{(p) r_{3}} \mathcal{N}_{s_{1} s_{2}}^{(p+2) s_{3}} \\
& \times\left(\frac{\delta_{2}(k+l)}{\left|z_{12}\right|^{h_{1}+h_{2}-h_{3}}} D_{\left(r_{1} s_{1}\right)\left(r_{2} s_{2}\right)}^{\mathrm{NS}}{ }^{\left(r_{3} s_{3}\right)} \phi_{r_{3} s_{3}}\left(z_{2}\right)\right. \\
& \left.\quad+\frac{\delta_{2}(k+l+1)}{\left|z_{12}\right|^{h_{1}+h_{2}-\tilde{h}_{3}}} \tilde{D}_{\left(r_{1} s_{1}\right)\left(r_{2} s_{2}\right)}^{\mathrm{NS}}\left(r_{3} s_{3}\right) \tilde{\phi}_{r_{3} s_{3}}\left(z_{2}\right)\right),
\end{aligned}
$$

where $k=\frac{r_{1}+r_{2}-r_{3}+1}{2}, l=\frac{s_{1}+s_{2}-s_{3}+1}{2}$. We used the notation $\delta_{2}(m)$ for a function that is 1 if $m$ is even, and 0 if it is odd. The conformal weights of the fields are denoted by $h_{i}=h_{r_{i} s_{i}}^{\mathrm{NS}}$ with

$$
h_{r s}^{\mathrm{NS}}=\frac{((p+2) r-p s)^{2}-4}{8 p(p+2)} .
$$

The field $\tilde{\phi}_{r s}$ denotes the superpartner to the field $\phi_{r s}$ with conformal weight $\tilde{h}_{r s}^{\mathrm{NS}}$.

As in the case of Virasoro minimal models, it is more convenient to state the structure constants $C, \tilde{C}$ for a different normalisation of the fields. The normalised structure constants are recovered again by (A.5). These constants have been determined in 14 and can be written as

$$
\begin{aligned}
C_{\left(r_{1} s_{1}\right)\left(r_{2} s_{2}\right)}^{\mathrm{NS}}\left(r_{3} s_{3}\right)= & \prod_{x=0,-d_{1},-d_{2}, d_{3}}\left(\prod_{\substack{n=1 \\
m+n \text { even }}}^{l-1} \prod_{m=1}^{k-1} \frac{t}{\left(\frac{d_{m n}+x}{2}\right)^{2}}\right. \\
& \left.\times \prod_{m=1}^{k-1} \frac{\Gamma\left(\frac{x+m+t \delta_{2}(m+1)}{2 t}\right)}{\Gamma\left(1-\frac{x+m+t \delta_{2}(m+1)}{2 t}\right)} \prod_{n=1}^{l-1} \frac{\Gamma\left(\frac{n t-x+\delta_{2}(n+1)}{2}\right)}{\Gamma\left(1-\frac{n t-x+\delta_{2}(n+1)}{2}\right)}\right),
\end{aligned}
$$

and

$$
\tilde{C}_{\left(r_{1} s_{1}\right)\left(r_{2} s_{2}\right)}^{\mathrm{NS}}\left(r_{3} s_{3}\right)=C_{\left(r_{1} s_{1}\right)\left(r_{2} s_{2}\right)}^{\mathrm{NS}}\left(r_{3} s_{3}\right) \cdot \begin{cases}\frac{2}{\left(\frac{r_{3}+1}{2 t}-\frac{s_{3}+1}{2}\right)^{2}}, & k \text { even, } l \text { odd } \\ \frac{t^{2}\left(\frac{r_{3}+1}{2 t}-\frac{s_{3}+1}{2}\right)^{2}}{2} & k \text { odd, } l \text { even } .\end{cases}
$$

Here, $d_{m n}=m-n t, d_{i}=d_{r_{i} s_{i}}$ and $t=\frac{p}{p+2}$.

Let us now try to find an expression for the structure constants that only depends on the variables $d_{i}$. The strategy is similar to the one pursued in the case of Virasoro minimal models in the previous section. Let us first rewrite the double product in the first line 
of (A.21) as

$$
\begin{aligned}
& \prod_{\substack{n=1 \\
m+n \text { even }}}^{l-1} \prod_{m=1}^{k-1} \frac{t}{\left(\frac{d_{m n}+x}{2}\right)^{2}}=(-t)^{\left\lfloor\frac{k}{2}\right\rfloor\left\lfloor\frac{l}{2}\right\rfloor+\left\lfloor\frac{k-1}{2}\right\rfloor\left\lfloor\frac{l-1}{2}\right\rfloor} \\
& \times \prod_{n=1}^{l-1} \frac{\Gamma\left(1-\frac{n t-x+\delta_{2}(n+1)}{2}\right)}{\Gamma\left(\frac{x-n t+k+1-\delta_{2}(k+n)}{2}\right)} \frac{\Gamma\left(1-\frac{x-n t+k+1-\delta_{2}(k+n)}{2}\right)}{\Gamma\left(\frac{n t-x+\delta_{2}(n+1)}{2}\right)} .
\end{aligned}
$$

The structure constants then become

$$
\begin{aligned}
C_{\left(r_{1} s_{1}\right)\left(r_{2} s_{2}\right)}^{\mathrm{NS}}\left(r_{3} s_{3}\right)= & \left(t^{4}\right)^{\left\lfloor\frac{k}{2}\right\rfloor\left\lfloor\frac{l}{2}\right\rfloor+\left\lfloor\frac{k-1}{2}\right\rfloor\left\lfloor\frac{l-1}{2}\right\rfloor .} \prod_{x=0,-d_{1},-d_{2}, d_{3}} \\
& \times\left(\prod_{m=1}^{k-1} \frac{\Gamma\left(\frac{x+m+t \delta_{2}(m+1)}{2 t}\right)}{\Gamma\left(1-\frac{x+m+t \delta_{2}(m+1)}{2 t}\right)} \prod_{n=1}^{l-1} \frac{\Gamma\left(1-\frac{x-n t+k+1-\delta_{2}(k+n)}{2}\right)}{\Gamma\left(\frac{x-n t+k+1-\delta_{2}(k+n)}{2}\right)}\right) .
\end{aligned}
$$

Now we replace the products of gamma functions by ratios of Barnes' double gamma functions. Take the first product over $m$. We split it up into two products, one over even $m$ and one over odd $m$. Employing the formula (A.11) for the separate products then yields

$$
\begin{aligned}
\prod_{m=1}^{k-1} \frac{\Gamma\left(\frac{x+m+t \delta_{2}(m+1)}{2 t}\right)}{\Gamma\left(1-\frac{x+m+t \delta_{2}(m+1)}{2 t}\right)}= & t^{-\frac{1}{2 t}\left\lfloor\frac{k}{2}\right\rfloor\left(x+\left\lfloor\frac{k}{2}\right\rfloor\right)} \cdot t^{-\frac{1}{2 t}\left\lfloor\frac{k-1}{2}\right\rfloor\left(x+1-t+\left\lfloor\frac{k-1}{2}\right\rfloor\right)} \\
& \times \frac{\Upsilon\left(\left(\frac{x+1+t}{2}+\left\lfloor\frac{k}{2}\right\rfloor\right) t^{-\frac{1}{2}} \mid t^{-\frac{1}{2}}\right) \Upsilon\left(\left(\frac{x+2}{2}+\left\lfloor\frac{k-1}{2}\right\rfloor\right) t^{-\frac{1}{2}} \mid t^{-\frac{1}{2}}\right)}{\Upsilon\left(\frac{x+1+t}{2} t^{-\frac{1}{2}} \mid t^{-\frac{1}{2}}\right) \Upsilon\left(\frac{x+2}{2} t^{-\frac{1}{2}} \mid t^{-\frac{1}{2}}\right)}
\end{aligned}
$$

Doing the same with the product over $n$ leads to

$$
\begin{aligned}
& \prod_{n=1}^{l-1} \frac{\Gamma\left(1-\frac{x-n t+k+1-\delta_{2}(k+n)}{2}\right)}{\Gamma\left(\frac{x-n t+k+1-\delta_{2}(k+n)}{2}\right)}=t^{-\left\lfloor\frac{l}{2}\right\rfloor\left(\frac{x}{2}+\left\lfloor\frac{k}{2}\right\rfloor-\frac{t}{2}\left\lfloor\frac{l}{2}\right\rfloor\right)} \cdot t^{-\left\lfloor\frac{l-1}{2}\right\rfloor\left(\frac{x-t+1}{2}+\left\lfloor\frac{k-1}{2}\right\rfloor-\frac{t}{2}\left\lfloor\frac{l-1}{2}\right\rfloor\right)} \\
& \times \frac{\Upsilon\left(\left(\frac{x+1+t}{2}+\left\lfloor\frac{k}{2}\right\rfloor-t\left\lfloor\frac{l}{2}\right\rfloor\right) t^{-\frac{1}{2}} \mid t^{-\frac{1}{2}}\right) \Upsilon\left(\left(\frac{x+2}{2}+\left\lfloor\frac{k-1}{2}\right\rfloor-t\left\lfloor\frac{l-1}{2}\right\rfloor\right) t^{-\frac{1}{2}} \mid t^{-\frac{1}{2}}\right)}{\Upsilon\left(\left(\frac{x+1+t}{2}+\left\lfloor\frac{k}{2}\right\rfloor\right) t^{-\frac{1}{2}} \mid t^{-\frac{1}{2}}\right) \Upsilon\left(\left(\frac{x+2}{2}+\left\lfloor\frac{k-1}{2}\right\rfloor\right) t^{-\frac{1}{2}} \mid t^{-\frac{1}{2}}\right)} .
\end{aligned}
$$

When we combine the results, we obtain

$$
\begin{aligned}
& C_{\left(r_{1} s_{1}\right)\left(r_{2} s_{2}\right)}^{\mathrm{NS}}{ }^{\left(r_{3} s_{3}\right)}=t^{-(1-t)\left(\frac{k-1}{2 t}+\frac{l-1}{2}\right)+\delta_{2}(l)-\delta_{2}(k)} \\
& \times \prod_{x=0,-d_{1},-d_{2}, d_{3}} \frac{\Upsilon\left(\left(\frac{x+1+t+d_{k l}}{2}+\frac{t \delta_{2}(k+l+1)}{2}\right) t^{-\frac{1}{2}} \mid t^{-\frac{1}{2}}\right) \Upsilon\left(\left(\frac{x+2 t+d_{k l}}{2}-\frac{t \delta_{2}(k+l+1)}{2}\right) t^{-\frac{1}{2}} \mid t^{-\frac{1}{2}}\right)}{\Upsilon\left(\frac{x+1+t}{2} t^{-\frac{1}{2}} \mid t^{-\frac{1}{2}}\right) \Upsilon\left(\frac{x+2}{2} t^{-\frac{1}{2}} \mid t^{-\frac{1}{2}}\right)} .
\end{aligned}
$$

Except for the exponent of $t$ we get a result that only depends on the variables $d_{i}$ and on $k+l$ being even or odd, corresponding to even or odd fusion. 
To obtain the normalised structure constants $D^{\mathrm{NS}}$ we first determine

$$
C_{(r s)(r s)}^{\mathrm{NS}}(11)=t^{-(1-t)\left(\frac{r-1}{2 t}+\frac{s-1}{2}\right)} \frac{\Upsilon\left(\frac{2+d_{r s}}{2} t^{-\frac{1}{2}} \mid t^{-\frac{1}{2}}\right) \Upsilon\left(\frac{1+t}{2} t^{-\frac{1}{2}} \mid t^{-\frac{1}{2}}\right)}{\Upsilon\left(\frac{2 t+d_{r s}}{2} t^{-\frac{1}{2}} \mid t^{-\frac{1}{2}}\right) \Upsilon\left(\frac{3-t}{2} t^{-\frac{1}{2}} \mid t^{-\frac{1}{2}}\right)} .
$$

The normalised structure constants are then given by

$$
D_{\left(r_{1} s_{1}\right)\left(r_{2} s_{2}\right)}^{\mathrm{NS}}\left(r_{3} s_{3}\right)=D_{0}^{\mathrm{NS}} \prod_{\substack{x=0,-d_{1},-d_{2}, d_{3}}} \frac{\Upsilon\left(\frac{x+1+t+d_{k l}}{2} t^{-\frac{1}{2}} \mid t^{-\frac{1}{2}}\right) \Upsilon\left(\frac{x+2 t+d_{k l}}{2} t^{-\frac{1}{2}} \mid t^{-\frac{1}{2}}\right)}{\Upsilon\left(\frac{x+1+t}{2} t^{-\frac{1}{2}} \mid t^{-\frac{1}{2}}\right)\left[\Upsilon\left(\frac{x+2}{2} t^{-\frac{1}{2}} \mid t^{-\frac{1}{2}}\right) \Upsilon\left(\frac{x+2 t}{2} t^{-\frac{1}{2}} \mid t^{-\frac{1}{2}}\right)\right]^{\frac{1}{2}}}
$$

with the normalisation constant

$$
D_{0}^{\mathrm{NS}}=t^{-\frac{1}{4}\left(t-t^{-1}+2\right)}\left[\gamma\left(\frac{t+1}{2}\right) \gamma\left(\frac{t^{-1}-1}{2}\right)\right]^{\frac{1}{2}} .
$$

The structure constants (A.29) are invariant under permutation of the field labels, and do not change if one applies a field identification to two of the field labels, e.g. $d_{1} \rightarrow-d_{1}$ and $d_{2} \rightarrow-d_{2}$. The structure constants for the OPE involving superdescendants are given by

$$
\tilde{D}_{\left(r_{1} s_{1}\right)\left(r_{2} s_{2}\right)}^{\mathrm{NS}}\left(r_{3} s_{3}\right)=\frac{8 t D_{0}^{\mathrm{NS}}}{\left(d_{3}+1-t\right)^{2}} \prod_{\substack{x=0,-d_{1} \\-d_{2}, d_{3}}} \frac{\Upsilon\left(\frac{x+1+2 t+d_{k l}}{2} t^{-\frac{1}{2}} \mid t^{-\frac{1}{2}}\right) \Upsilon\left(\frac{x+t+d_{k l}}{2} t^{-\frac{1}{2}} \mid t^{-\frac{1}{2}}\right)}{\Upsilon\left(\frac{x+1+t}{2} t^{-\frac{1}{2}} \mid t^{-\frac{1}{2}}\right)\left[\Upsilon\left(\frac{x+2}{2} t^{-\frac{1}{2}} \mid t^{-\frac{1}{2}}\right) \Upsilon\left(\frac{x+2 t}{2} t^{-\frac{1}{2}} \mid t^{-\frac{1}{2}}\right)\right]^{\frac{1}{2}}} .
$$

Similar to the discussion at the end of appendix A.1, given the structure constants for all superconformal minimal models with rational $t$, the provided expressions (A.29), (A.31) give the unique interpolation that is continuous both in $d$ and in $t$.

\section{B. Special functions}

Barnes' double gamma function $\Gamma_{2}\left(x \mid b, b^{-1}\right)$ is defined for $x \in \mathbb{C}$ and complex $b$ with $\operatorname{Re} b \neq 0$ (see 49]), and can be written as

$$
\log \Gamma_{2}\left(x \mid b, b^{-1}\right)=\left(\frac{\partial}{\partial t} \sum_{n_{1}, n_{2}=0}^{\infty}\left(x+n_{1} b+n_{2} b^{-1}\right)^{-t}\right)_{t=0} .
$$

We shall work with a different normalisation, $\Gamma_{2}(x \mid b):=\Gamma_{2}\left(x \mid b, b^{-1}\right) / \Gamma_{2}\left(Q / 2 \mid b, b^{-1}\right)$. The logarithm of $\Gamma_{2}$ can be represented by an integral,

$$
\log \Gamma_{2}(x \mid b)=-\int_{0}^{\infty} \frac{\mathrm{d} t}{t}\left(\frac{e^{-Q t / 2}-e^{-x t}}{\left(1-e^{-b t}\right)\left(1-e^{-t / b}\right)}+\frac{(Q / 2-x)^{2}}{2} e^{-t}+\frac{Q / 2-x}{t}\right),
$$

where $Q=b+b^{-1}$. The integral converges for $x$ with $\operatorname{Re} x>0$.

The double gamma function satisfies the functional relations

$$
\begin{gathered}
\Gamma_{2}(x+b \mid b)=\frac{\sqrt{2 \pi}}{\Gamma(b x)} b^{-1 / 2+b x} \Gamma_{2}(x \mid b) \\
\Gamma_{2}(x+1 / b \mid b)=\frac{\sqrt{2 \pi}}{\Gamma(x / b)} b^{-x / b+1 / 2} \Gamma_{2}(x \mid b) .
\end{gathered}
$$


Further properties of the double gamma function can be found in the literature (see e.g. [50]). We also need the combination

$$
\Upsilon(x \mid b)=\Gamma_{2}^{-1}(x \mid b) \Gamma_{2}^{-1}(Q-x \mid b) .
$$

The function $\Upsilon$ also has a simple behaviour under shifts of the argument by $b$ or $b^{-1}$,

$$
\begin{aligned}
\Upsilon(x+b \mid b) & =\gamma(b x) b^{1-2 b x} \Upsilon(x \mid b) \\
\Upsilon\left(x+b^{-1} \mid b\right) & =\gamma\left(b^{-1} x\right) b^{-1+2 x b^{-1}} \Upsilon(x \mid b),
\end{aligned}
$$

where $\gamma(x)=\frac{\Gamma(x)}{\Gamma(1-x)}$.

\section{Fusion rules from Liouville}

In the $b \rightarrow i$ limit of Liouville theory, the fusion rules of the limiting theory at $c=\frac{3}{2}$ arise from the term $e^{-\frac{2}{\epsilon} F\left(\frac{p_{i}}{2}\right)}$ in the limit $\epsilon \rightarrow 0$, with the function $F$ (see (3.17)) given by

$$
F\left(\frac{p_{i}}{2}\right)=\sum_{i=1}^{3}\left(\lambda\left(p_{i}\right)-\lambda\left(\tilde{p}_{i}\right)\right)-\lambda(\tilde{p})+\frac{\pi^{2}}{2} .
$$

The function $\lambda$ is given after (3.12). Here we want to prove that $F$ is always greater or equal to zero (so the limit of $e^{-\frac{2}{\epsilon} F}$ is well defined), in particular

$$
F\left(\frac{p_{i}}{2}\right)= \begin{cases}0 \quad\left\lfloor p_{1}\right\rfloor+\left\lfloor p_{2}\right\rfloor+\left\lfloor p_{3}\right\rfloor \text { even } \\ \text { and }\left|\left\{p_{1}\right\}-\left\{p_{2}\right\}\right| \leq\left\{p_{3}\right\} \leq \min \left(\left\{p_{1}\right\}+\left\{p_{2}\right\}, 2-\left\{p_{1}\right\}-\left\{p_{2}\right\}\right) \\ 0 \quad\left\lfloor p_{1}\right\rfloor+\left\lfloor p_{2}\right\rfloor+\left\lfloor p_{3}\right\rfloor \text { odd } \\ \text { and }\left|\left\{p_{1}\right\}-\left\{p_{2}\right\}\right| \leq 1-\left\{p_{3}\right\} \leq \min \left(\left\{p_{1}\right\}+\left\{p_{2}\right\}, 2-\left\{p_{1}\right\}-\left\{p_{2}\right\}\right) \\ \text { positive otherwise. }\end{cases}
$$

To study the function $F$ we have to relate the fractional part of $\tilde{p}$ and $\tilde{p}_{i}$ to the fractional parts $\left\{p_{i}\right\}$. We first look at the case when $\left\lfloor p_{1}\right\rfloor+\left\lfloor p_{2}\right\rfloor+\left\lfloor p_{3}\right\rfloor$ is even. We find

$$
\begin{gathered}
\{\tilde{p}\}=\left\{\frac{\left\{p_{1}\right\}+\left\{p_{2}\right\}+\left\{p_{3}\right\}}{2}\right\}= \begin{cases}\frac{\left\{p_{1}\right\}+\left\{p_{2}\right\}+\left\{p_{3}\right\}}{2}, & \left\{p_{3}\right\}<2-\left\{p_{1}\right\}-\left\{p_{2}\right\} \\
\frac{\left\{p_{1}\right\}+\left\{p_{2}\right\}+\left\{p_{3}\right\}}{2}-1, & \left\{p_{3}\right\} \geq 2-\left\{p_{1}\right\}-\left\{p_{2}\right\}\end{cases} \\
\left\{\tilde{p}_{i}\right\}=\left\{\frac{\sum_{j}\left\{p_{j}\right\}-2\left\{p_{i}\right\}}{2}\right\}=\left\{\begin{array}{ll}
\frac{\sum_{j}\left\{p_{j}\right\}-2\left\{p_{i}\right\}}{2}, & 2\left\{p_{i}\right\} \leq \sum_{j}\left\{p_{j}\right\} \\
\frac{\sum_{j}\left\{p_{j}\right\}-2\left\{p_{i}\right\}}{2}+1, & 2\left\{p_{i}\right\}>\sum_{j}\left\{p_{j}\right\}
\end{array} .\right.
\end{gathered}
$$

Evaluating $\lambda(\tilde{p}), \lambda\left(\tilde{p}_{i}\right)$ we get

$$
\begin{aligned}
\lambda(\tilde{p}) & = \begin{cases}\frac{\pi^{2}}{2}\left(\sum_{j}\left\{p_{j}\right\}-1\right)^{2}, & \left\{p_{3}\right\} \leq 2-\left\{p_{1}\right\}-\left\{p_{2}\right\} \\
\frac{\pi^{2}}{2}\left(\sum_{j}\left\{p_{j}\right\}-1\right)^{2}-2 \pi^{2}\left(\sum_{j}\left\{p_{j}\right\}-2\right), & \left\{p_{3}\right\}>2-\left\{p_{1}\right\}-\left\{p_{2}\right\}\end{cases} \\
\lambda\left(\tilde{p}_{i}\right) & =\left\{\begin{array}{lr}
\frac{\pi^{2}}{2}\left(\sum_{j}\left\{p_{j}\right\}-2\left\{p_{i}\right\}-1\right)^{2}, & 2\left\{p_{i}\right\} \leq \sum_{j}\left\{p_{j}\right\} \\
\frac{\pi^{2}}{2}\left(\sum_{j}\left\{p_{j}\right\}-2\left\{p_{i}\right\}-1\right)^{2}-2 \pi^{2}\left(2\left\{p_{i}\right\}-\sum_{j}\left\{p_{j}\right\}\right), & 2\left\{p_{i}\right\}>\sum_{j}\left\{p_{j}\right\} .
\end{array}\right.
\end{aligned}
$$


Let us consider the case when the first lines of (C.5) and (C.6) apply. A straightforward calculation shows that $F=0$. This corresponds to the first case in (C.2). It is easy to see that in any other case, the function $F$ obtains an additional term that makes it positive. This concludes the case $\sum_{j}\left\lfloor p_{j}\right\rfloor$ even.

If $\sum_{j}\left\lfloor p_{j}\right\rfloor$ is odd, we can still use the analysis above if we replace $p_{i} \rightarrow-p_{i}$. The function $F$ is invariant under this replacement, and for non-integer $p_{i}$ we have $\left\lfloor-p_{i}\right\rfloor=$ $-\left\lfloor p_{i}\right\rfloor-1$, so for generic numbers $p_{i}$ the sum $\sum_{i}\left\lfloor-p_{i}\right\rfloor$ is again even and we are back to the case we considered before. The fractional parts are then related by $\left\{-p_{i}\right\}=1-\left\{p_{i}\right\}$, and in this way we obtain the second line of the result for $F$ in (C.2) from the first line. If one or more labels $p_{i}$ are integer, the same arguments apply: we can still replace $\left\{p_{i}\right\}$ by $1-\left\{p_{i}\right\}$ (which maps 0 to 1 ) to relate the problem to the old analysis (we there did not use the fact that the fractional parts were strictly less than 1 ).

\section{References}

[1] A. Cappelli, C. Itzykson and J.B. Zuber, The ADE classification of minimal and A1(1) conformal invariant theories, Commun. Math. Phys. 113 (1987) 1.

[2] A. Kato, Classification of modular invariant partition functions in two dimensions, Mod. Phys. Lett. A 2 (1987) 585.

[3] P.H. Ginsparg, Curiosities at $c=1$, Nucl. Phys. B 295 (1988) 153.

[4] E.B. Kiritsis, Proof of the completeness of the classification of rational conformal theories with $c=1$, Phys. Lett. B 217 (1989) 427.

[5] I. Runkel and G.M.T. Watts, A non-rational CFT with $c=1$ as a limit of minimal models, JHEP 09 (2001) 006 hep-th/0107118.

[6] V. Schomerus, Rolling tachyons from Liouville theory, JHEP 11 (2003) 043 hep-th/0306026.

[7] K. Graham, I. Runkel and G.M.T. Watts, Minimal model boundary flows and $c=1$ CFT, Nucl. Phys. B 608 (2001) 527 hep-th/0101187.

[8] S. Fredenhagen and V. Schomerus, Boundary Liouville theory at $c=1$, JHEP 05 (2005) 025 hep-th/0409256.

[9] A.B. Zamolodchikov and A.B. Zamolodchikov, Liouville field theory on a pseudosphere, hep-th/0101152.

[10] V. Fateev, A.B. Zamolodchikov and A.B. Zamolodchikov, Boundary Liouville field theory. I: boundary state and boundary two-point function, hep-th/0001012.

[11] J. Teschner, Remarks on Liouville theory with boundary, hep-th/0009138.

[12] A. Cappelli, Modular invariant partition functions of superconformal theories, Phys. Lett. B $185(1987) 82$.

[13] L.J. Dixon, P.H. Ginsparg and J.A. Harvey, $\hat{c}=1$ superconformal field theory, Nucl. Phys. B $306(1988) 470$.

[14] Y. Kitazawa et al., Operator product expansion coefficients in $N=1$ superconformal theory and slightly relevant perturbation, Nucl. Phys. B 306 (1988) 425. 
[15] L. Álvarez-Gaumé and P. Zaugg, Structure constants in the $N=1$ superoperator algebra, Ann. Phys. (NY) 215 (1992) 171 hep-th/9109050.

[16] R.H. Poghosian, Structure constants in the $N=1$ super-Liouville field theory, Nucl. Phys. B 496 (1997) 451 hep-th/9607120.

[17] R.C. Rashkov and M. Stanishkov, Three-point correlation functions in $N=1$ super Liouville theory, Phys. Lett. B 380 (1996) 49 hep-th/9602148.

[18] T. Fukuda and K. Hosomichi, Super Liouville theory with boundary, Nucl. Phys. B 635 (2002) 215 hep-th/0202032.

[19] A. Belavin, V. Belavin, A. Neveu and A. Zamolodchikov, Bootstrap in supersymmetric Liouville field theory. I: NS sector, hep-th/0703084.

[20] D. Friedan, Z.-a. Qiu and S.H. Shenker, Conformal invariance, unitarity and two-dimensional critical exponents, Phys. Rev. Lett. 52 (1984) 1575.

[21] J. Rasmussen, Logarithmic limits of minimal models, Nucl. Phys. B 701 (2004) 516 hep-th/0405257.

[22] R.I. Nepomechie, Consistent superconformal boundary states, J. Phys. A 34 (2001) 6509 hep-th/0102010.

[23] A.M. Polyakov, Quantum geometry of fermionic strings, Phys. Lett. B 103 (1981) 211.

[24] H. Dorn and H.J. Otto, Two and three point functions in Liouville theory, Nucl. Phys. B 429 (1994) 375 hep-th/9403141.

[25] A.B. Zamolodchikov and A.B. Zamolodchikov, Structure constants and conformal bootstrap in Liouville field theory, Nucl. Phys. B 477 (1996) 577 hep-th/9506136.

[26] P.H. Ginsparg, Applied conformal field theory, hep-th/9108028.

[27] C. Ahn, C. Rim and M. Stanishkov, Exact one-point function of $N=1$ super-Liouville theory with boundary, Nucl. Phys. B 636 (2002) 497 hep-th/0202043.

[28] L. Hadasz, Z. Jaskolski and P. Suchanek, Recursion representation of the Neveu-Schwarz superconformal block, JHEP 03 (2007) 032 hep-th/0611266].

[29] V.A. Belavin, $N=1$ SUSY conformal block recursive relations, hep-th/0611295.

[30] G. Mussardo, G. Sotkov and M. Stanishkov, Ramond sector of the supersymmetric minimal models, Phys. Lett. B 195 (1987) 397.

[31] G. Mussardo, G. Sotkov and H. Stanishkov, Fine structure of the supersymmetric operator product expansion algebras, Nucl. Phys. B 305 (1988) 69.

[32] A.B. Zamolodchikov and V.A. Fateev, Operator algebra and correlation functions in the two-dimensional Wess-Zumino $\mathrm{SU}(2) \times \mathrm{SU}(2)$ chiral model, Sov. J. Nucl. Phys. 43 (1986) 657 .

[33] P. Christe and R. Flume, The four point correlations of all primary operators of the $D=2$ conformally invariant $\mathrm{SU}(2)$ sigma model with Wess-Zumino term, Nucl. Phys. B 282 (1987) 466.

[34] G. Mussardo, G. Sotkov and M. Stanishkov, $N=2$ superconformal minimal models, Int. J. Mod. Phys. A 4 (1989) 1135.

[35] A. Dabholkar and A. Pakman, Exact chiral ring of $A d S_{3} / C F T_{2}$, hep-th/0703022. 
[36] G. D'Appollonio and E. Kiritsis, String interactions in gravitational wave backgrounds, Nucl. Phys. B 674 (2003) 80 hep-th/0305081.

[37] K. Hosomichi, $N=2$ Liouville theory with boundary, JHEP 12 (2006) 061 hep-th/0408172.

[38] A. Giveon and D. Kutasov, Little string theory in a double scaling limit, JHEP 10 (1999) 034 hep-th/9909110.

[39] J. Teschner, On structure constants and fusion rules in the $\mathrm{SL}(2, \mathbb{C}) / \mathrm{SU}(2)$ WZNW model, Nucl. Phys. B 546 (1999) 390 hep-th/9712256.

[40] V.A. Fateev and A.V. Litvinov, On differential equation on four-point correlation function in the conformal Toda field theory, JETP Lett. 81 (2005) 594 hep-th/0505120.

[41] S.L. Lukyanov and V.A. Fateev, Physics reviews: additional symmetries and exactly soluble models in two-dimensional conformal field theory, Chur, Harwood, Switzerland (1990).

[42] W. McElgin, Notes on Liouville theory at $c \leq 1$, arXiv:0706.0365.

[43] A.B. Zamolodchikov, Three-point function in the minimal Liouville gravity, Theor. Math. Phys. 142 (2005) 183.

[44] A.A. Belavin and A.B. Zamolodchikov, Integrals over moduli spaces, ground ring, and four-point function in minimal Liouville gravity, Theor. Math. Phys. 147 (2006) 729.

[45] D. Wellig, The limit of $N=1$ minimal models, Diploma thesis, ETH Zürich (2006).

[46] V.S. Dotsenko and V.A. Fateev, Conformal algebra and multipoint correlation functions in $2 D$ statistical models, Nucl. Phys. B 240 (1984) 312.

[47] V.S. Dotsenko and V.A. Fateev, Four point correlation functions and the operator algebra in the two-dimensional conformal invariant theories with the central charge $c<1$, Nucl. Phys. B 251 (1985) 691.

[48] V.S. Dotsenko and V.A. Fateev, Operator algebra of two-dimensional conformal theories with central charge $c \leq 1$, Phys. Lett. B 154 (1985) 291.

[49] E. Barnes, The theory of the double gamma function, Philos. Trans. Roy. Soc. A 196 (1901) 265.

[50] M. Jimbo and T. Miwa, $Q K Z$ equation with $|q|=1$ and correlation functions of the $X X Z$ model in the gapless regime, J. Phys. A 29 (1996) 2923 hep-th/9601135. 